\title{
Temperature dependence of transport coefficients of QCD in high-energy heavy-ion collisions
}

\author{
Kazuhisa Okamoto1* and Chiho Nonaka ${ }^{1,2}$ 计 \\ ${ }^{1}$ Department of Physics, Nagoya University, Nagoya 464-8602, Japan \\ ${ }^{2}$ Kobayashi Maskawa Institute, Nagoya University, Nagoya 464-8602, Japan
}

(Dated: January 8, 2019)

\begin{abstract}
Using our developed new relativistic viscous hydrodynamics code, we investigate the temperature dependence of shear and bulk viscosities from comparison with the ALICE data: single particle spectra and collective flows of $\mathrm{Pb}+\mathrm{Pb} \sqrt{s_{\mathrm{NN}}}=2.76 \mathrm{TeV}$ collisions at the Large Hadron Collider. We find that from the comprehensive analyses of centrality dependence of single particle spectra and collective flows, we can extract detailed information on the quark-gluon plasma bulk property, without the information being smeared by the final state interactions.
\end{abstract}

\section{INTRODUCTION}

Since the success of production of strongly interacting quark-gluon plasma (QGP) at the Relativistic Heavy Ion Collider (RHIC) [1], a relativistic viscous hydrodynamic model has been widely used for the description of spacetime evolution of the hot and dense matter created after collisions. Now at RHIC as well as at the Large Hadron Collider (LHC) high-energy heavy-ion collisions are performed and many experimental data are reported. Because the relativistic viscous hydrodynamic equation has close a relation to an equation of state (EoS) and transport coefficients of the QCD matter, analyses of experimental data at RHIC and the LHC based on a relativistic viscous hydrodynamic model can provide an insight into the detailed information on the QGP bulk property.

The recent development of a lattice QCD calculation for the EoS at vanishing chemical potential is remarkable. Two groups, the Wuppertal-Budapest and HotQCD Collaborations, report almost the same (pseudo)critical temperatures, $T_{c}=155 \pm 6 \mathrm{MeV}$ [2] and $T_{c}=154 \pm 9$ $\mathrm{MeV}$ [3], respectively. On the other hand, the evaluation of the shear viscosity to entropy density ratio $\eta / s$ of the hadronic phase and the QGP phase is investigated based on the Boltzmann equation [4, 5]. Due to the existence of the Kovtun-Son-Starinets (KSS) bound, the lower bound of $\eta / s[6], \eta / s$ takes the minimum around the critical temperature [7]. On the other hand, the behavior of temperature dependence of the bulk viscosity to entropy density ratio is not clear. For example, $\zeta / s$ of a massive pion gas decreases with temperature [8, 9] below the critical temperature. Also, above the critical temperature, $\zeta / s$ of quark-gluon matter [10] or gluon plasma [11] decreases with temperature. There is not a conclusive understanding of quantitative information on the transport coefficients of QCD matter.

Therefore phenomenological analyses of transport coefficients from comparison with experimental data at RHIC and the LHC are indispensable [12 19]. It turns out that

\footnotetext{
* okamoto@hken.phys.nagoya-u.ac.jp

$\dagger$ nonaka@hken.phys.nagoya-u.ac.jp
}

the value of $\eta / s$ at the LHC is larger than that at RHIC, which suggests a temperature-dependent $\eta / s$. At RHIC elliptic flow $v_{2}$ is sensitive to the $\eta / s$ of the hadronic phase, whereas at the LHC it depends on $\eta / s$ of both the hadronic phase and the QGP phase [20 23]. Simultaneous analyses of $v_{2}$ at RHIC and the LHC give a constraint on the temperature dependence of $\eta / s[20,23$. Even at one collision energy, we can explore the temperature dependence of shear and bulk viscosities from centrality and/or rapidity dependence of observables. At peripheral collisions the effect of $\eta / s$ of the hadronic phase is dominant, compared with that of the QGP phase [21, 23]. At the forward rapidity where the temperature becomes small, the behavior of $\eta / s$ in the hadronic phase affects the elliptic flow [22, 24].

Now not only the shear viscosity but also the bulk viscosity are included in relativistic viscous hydrodynamic simulations [25 35]. Generally bulk viscosity reduces the growth of radial flow in hydrodynamic expansion. In computations with IP-Glasma initial condition, finite bulk viscosity is important for explanation of experimental data, for example, mean $p_{T}$ [32, 33, 35. However, the evaluation of the effect of bulk viscosity in the calculation of particle distribution in the Cooper-Frye formula is not fixed yet. Furthermore, from the application of Bayesian analyses to a model-to-data comparison, the temperature dependences of shear and bulk viscosities are investigated 34]. The results support that $\eta / s$ takes the minimum value around the critical temperature and increases with temperature in the QGP phase, and $\zeta / s$ takes the maximum value around the critical temperature.

Furthermore, to achieve the quantitative analyses of the transport coefficients of QCD matter from comparison with high statistics and high precision experimental data, we need to perform numerical calculations for relativistic viscous hydrodynamics with high accuracy. We have developed a new relativistic viscous hydrodynamics code optimized in the Milne coordinates [36]. The code is constructed based on a Riemann solver with the two shock approximation 37, 38. It is stable even with small numerical viscosity 39 .

Using our newly developed hydrodynamics code we investigate the temperature dependence of shear and bulk 
viscosities from comprehensive analyses of centrality and rapidity dependence of particle distributions and higher flow harmonics at the LHC.

This paper is organized as follows. We begin in Sec. II by showing the relativistic viscous hydrodynamic equations and the numerical algorithm for solving them briefly. In Sec. III we explain the phenomenological model: initial conditions, equation of states used in the relativistic viscous hydrodynamic equations, freezeout process, and final state interactions based on UrQMD. We discuss the temperature dependence of shear and bulk viscosities in Sec. IV] In Sec. V] we show our numerical results of particle distributions and collective flows at the LHC. We end in Sec. VI with our conclusions.

\section{RELATIVISTIC VISCOUS HYDRODYNAMIC EQUATION AND ALGORITHM}

In a hydrodynamic model, we numerically solve the relativistic viscous hydrodynamic equation which is based on the conservation equations, $T_{; \mu}^{\mu \nu}=0$, where $T^{\mu \nu}$ is the energy-momentum tensor. In the Landau frame, the energy-momentum tensor of the viscous fluid is decomposed as $T^{\mu \nu}=e u^{\mu} u^{\nu}-(p+\Pi) \Delta^{\mu \nu}+\pi^{\mu \nu}$, where $\Pi$ is the bulk pressure and $\pi^{\mu \nu}$ is the shear tensor [40]. The relativistic extension of Navier-Stokes theory in a nonrelativistic fluid usually is not an easy task because of a problem of acausality and instability [41-43]. The problem can be resolved by introducing the second-order terms of the viscous tensor and the derivative of fluid variables into the hydrodynamic equations [44, 45]. Recently turned out that the original Israel-Stewart theory [44, 45] does not reproduce the results of the kinetic equation quantitatively [46 49]. Here we use the relativistic viscous hydrodynamic equation derived from the Boltzmann equation based on the method of moments [50, 51]. The relaxation equations for the bulk viscous pressure $\Pi$ and the shear-stress tensor $\pi^{\mu \nu}$ read

$$
\begin{aligned}
\tau_{\Pi} \dot{\Pi}+\Pi= & -\zeta \theta-\delta_{\Pi \Pi} \Pi \theta+\lambda_{\Pi \pi} \pi^{\mu \nu} \sigma_{\mu \nu}, \\
\tau_{\pi} \dot{\pi}^{\langle\mu \nu\rangle}+\pi^{\mu \nu}= & 2 \eta \sigma^{\mu \nu}-\delta_{\pi \pi} \pi^{\mu \nu} \theta+\varphi_{7} \pi_{\alpha}^{\langle\mu} \pi^{\nu\rangle \alpha} \\
& -\tau_{\pi \pi} \pi_{\alpha}^{\langle\mu} \sigma^{\nu\rangle \alpha}+\lambda_{\pi \Pi} \Pi \sigma^{\mu \nu}
\end{aligned}
$$

where $\tau_{\Pi}$ and $\tau_{\pi}$ are the relaxation times and $\delta_{\Pi \Pi}, \lambda_{\Pi \pi}$, $\delta_{\pi \pi}, \varphi_{7}, \tau_{\tau \tau}$, and $\lambda_{\pi \Pi}$ are the transport coefficients. To analyze high-energy heavy-ion collisions where strong longitudinal expansion exists we perform numerical computation in the Milne coordinates [52]. For details, see Refs. [36, 38.

In our algorithm [36], we split the conservation equation into two parts, an ideal part and a viscous part using the Strang splitting method [53. It is also applied to evaluate the constitutive equations of the viscous tensors Eqs. (1) and (2). We decompose them into the following three parts, the convection equations, the relaxation equations, and the equations with source terms. In numerical simulation of relativistic hydrodynamic equation, a time-step size $\Delta \tau$ is usually determined by the CourantFriedrichs-Lewy (CFL) condition. However in the relativistic dissipative hydrodynamics, one needs to determine the value of $\Delta \tau$ carefully. To save computational cost, we use the piecewise exact solution (PES) method [37, instead of using a simple explicit scheme. If, however, the relaxation times are larger than $\Delta \tau$ determined by the CFL condition, the PES method is not applied. We have checked the energy and momentum conservation in a one-dimensional expansion of high-energy heavy-ion collisions [38] and the correctness of our code in the following test problems: the viscous Bjorken flow for onedimensional expansion and the Israel-Stewart theory in Gubser flow regime for the three-dimensional calculation [36.

\section{MODEL}

For an initial condition of our hydrodynamic model, we use $\mathrm{T}_{\mathrm{R}}$ ENTo [54, 55]. In the parametric model $\mathrm{T}_{\mathrm{R}}$ ENTo, the initial entropy density $s\left(\boldsymbol{x}, \eta_{s}\right)$ is given by a function on the transverse plane at midrapidity $f(\boldsymbol{x})$ and a rapidity-dependent function $g\left(\boldsymbol{x}, \eta_{s}\right) ; s\left(\boldsymbol{x}, \eta_{s}\right) \propto$ $f(\boldsymbol{x}) \times g\left(\boldsymbol{x}, \eta_{s}\right)$. The function $f(\boldsymbol{x})$ is given by

$$
f(\boldsymbol{x}) \propto\left(\frac{\tilde{T}_{A}^{p}+\tilde{T}_{B}^{p}}{2}\right)^{1 / p},
$$

where $\tilde{T}_{A}$ is the nucleus thickness function expressed by proton thickness function $T_{p}$,

$$
\begin{aligned}
& \tilde{T}_{A}(\boldsymbol{x})=\sum_{i=1}^{N_{\text {part }}} w_{i} T_{p}\left(\boldsymbol{x}-\boldsymbol{x}_{i}\right), \\
& T_{p}(\boldsymbol{x})=\frac{1}{2 \pi w^{2}} \exp \left(-\frac{\boldsymbol{x}^{2}}{2 w^{2}}\right) .
\end{aligned}
$$

$w_{i}$ is random weight for introduction of a negative binomial distribution of produced particles and $\omega$ is Gaussian nucleon width. A parameter $p$ in the function $f(\boldsymbol{x})$ can interpolate among different types of entropy schemes such as the wounded nucleon model $(p=1)$, KLN model $(p \sim-0.67)$, and IP-Glasma and EKRT $(p \sim 0)$ 55]. Throughout our calculations we fix the parameter $p$ to $p=0$, which is suggested by the Bayesian analyses [55]. The initial distribution in the rapidity direction is described by $g\left(\boldsymbol{x}, \eta_{s}\right)=g(\boldsymbol{x}, y) d y / d \eta_{s}$, where $g(\boldsymbol{x}, y)$ is constructed by the inverse Fourier transform of its cumulantgenerating function,

$$
\begin{aligned}
g(\boldsymbol{x}, y) & =\mathcal{F}^{-1}\{\tilde{g}(\boldsymbol{x}, k)\} \\
& =\mathcal{F}^{-1}\left\{\exp \left(i \mu k-\frac{1}{2} \sigma^{2} k^{2}-\frac{1}{6} \gamma \sigma^{3} k^{3}\right)\right\} .
\end{aligned}
$$

The first three cumulants $\mu, \sigma$, and $\gamma$ of the local rapidity distribution are parametrized by three corresponding 
coefficients $\mu_{0}, \sigma_{0}$, and $\gamma_{0}$ which characterize the rapidity distribution's shift, width, and skewness, respectively. $d y / d \eta_{s}$ is determined by the Jacobian parameter $J$. The initial entropy density contains the following parameters other than the parameter $p$ : normalization $N$, nucleon width $w$, multiplicity fluctuation shape $k$, rapidity distributions' shift $\mu_{0}$, width $\sigma_{0}$, and skewness $\gamma_{0}$, and Jacobian parameter $J$. We set initial flows and initial values of viscous tensors to be vanishing at $\tau_{0}=0.6 \mathrm{fm}$.

We use a realistic parametrized EoS [56] based on continuum-extrapolated lattice QCD results in the physical quark mass limit [57, which is also combined with a hadron resonance gas model [58, 59] at low temperature. In the parametrization, the sound velocity takes the minimum value at $T_{c} \sim 167 \mathrm{MeV}[56$.

At the switching temperature $T_{\mathrm{SW}}=150 \mathrm{MeV}$ the hydrodynamic expansion terminates and the UrQMD model starts for the description of space-time evolution 60. 61. We sample produced particles from the fluid, using the Cooper-Frye formula 62

$$
E \frac{d N_{i}}{d^{3} p}=\frac{g_{i}}{2 \pi} \int_{\Sigma} f_{i}(x, p) p^{\mu} d^{3} \sigma_{\mu}
$$

where $i$ is an index over particle species, $f_{i}$ is the distribution function, and $d^{3} \sigma_{\mu}$ is a volume element of the isothermal hypersurface $\Sigma$ defined by $T_{S W}$. We introduce the shear viscous correction to the distribution function based on Ref. 63. We neglect the bulk viscosity correction, because ambiguity in the estimate exists [30. Both bulk viscosity in the hydrodynamic expansion and the bulk viscous correction to the distribution function reduce the mean transverse momentum [26, 27, 29, 30, 32, 33, 64. We find the particlization hypersurface based on Ref. 65, checking the temperature of volume elements of the fluid at each time step. The sampled particle distribution is used for an initial state for UrQMD 66, 67]. In UrQMD all produced hadrons move along classical trajectories, including their scattering, resonance formations, and decay processes until interactions among them stop.

\section{TEMPERATURE-DEPENDENT TRANSPORT COEFFICIENTS}

One of the pioneer works of analyses of temperature dependent $\eta / s$ was done in Ref. [20]. More comprehensive analyses were performed in Refs. 22, 23. In Ref. 24] they authors showed that a temperature-independent $\eta / s$ is disfavored from comparison with experimental data at RHIC, and event-by-event flow as a function of rapidity is useful for constraining the temperature dependence of shear viscosity. The first analyses of experimental data with both shear and bulk viscosities of the hadronic phase were carried out in Ref. 28. The effect of bulk viscosity on higher flow harmonics was also investigated 30, 31. In addition, it was found that inclusion of bulk viscosity
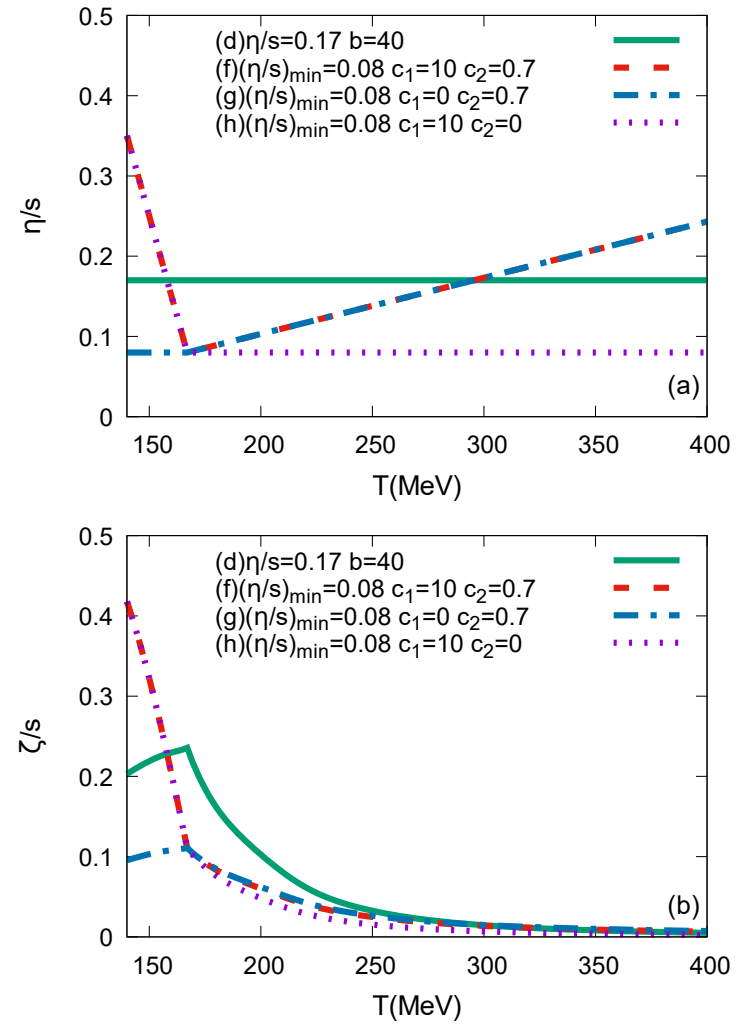

FIG. 1 (color online). The temperature dependence of the shear viscosity to entropy density ratio $\eta / s$ (a) and the bulk viscosity to entropy density ratio $\zeta / s(\mathrm{~b})$.

is preferable for a better description of the data of ultracentral relativistic heavy-ion collisions at the LHC [32].

Here we use the same parametrization as that in Refs. [51, 68, for bulk viscosity,

$$
\frac{\zeta}{s}=b \frac{\eta}{s}\left(\frac{1}{3}-c_{s}^{2}\right)^{2}
$$

where $c_{s}$ is the sound velocity and $b$ is a parameter. We parametrize $\eta / s(T)$ [23, 24],

$\frac{\eta}{s}(T)=\left(\frac{\eta}{s}\right)_{\text {min }}+c_{1}\left(T_{c}-T\right) \theta\left(T_{c}-T\right)+c_{2}\left(T-T_{c}\right) \theta\left(T-T_{c}\right)$,

where $c_{1}$ and $c_{2}$ are parameters and $T_{c}=167 \mathrm{MeV}$. We compare our calculated results with experimental data, in the case of the following parameter sets (Fig. 1): (d) $\eta / s=0.17, b=40 ;$ (f) $(\eta / s)_{\min }=0.08, c_{1}=10, c_{2}=$ $0.7, b=40 ;(\mathrm{g})(\eta / s)_{\min }=0.08, c_{1}=0, c_{2}=0.7, b=40$; and $(\mathrm{h})(\eta / s)_{\min }=0.08, c_{1}=10, c_{2}=0, b=40$. The values of $c_{1}$ and $c_{2}$ are given in $\mathrm{GeV}^{-1}$. Because the speed of sound takes the minimum value at $T_{c}$ [56], the temperature dependence of bulk viscosity has a sharp peak at $T_{c}$ in the case of $(\mathrm{d})$. 


\section{NUMERICAL RESULTS}

\section{A. Parameters in computations}

In the next subsections we explore transport coefficients of QGP through quantitative analyses of the LHC data, such as one-particle disaributions and collective flows in $\mathrm{Pb}+\mathrm{Pb} \sqrt{s_{N N}}=2.76 \mathrm{TeV}$ collisions. First in Sec. VB we discuss an appropriate value of constant shear viscosity without including bulk viscosity and then in the Sec. VC we consider the effect of bulk viscosity on physical observables. In Sec. VD we investigate the temperature dependence of the shear viscosity. Finally in Sec. VE, we discuss the effect of final state interactions to $p_{T}$ spectra and collective flows. We determine parameters in the initial condition $\mathrm{T}_{\mathrm{R}} \mathrm{ENT}$, using pseudorapidity distributions of charged hadrons at central collision 69, 70. Our parameters are the same as those in Ref. 55], except for the normalization $N$ and $\sigma_{0}$.

We fix the centrality based on initial entropy densities. First we produce initial entropy distributions of a certain number of events with minimum bias, using $\mathrm{T}_{\mathrm{R}}$ ENTo. Then we arrange the events in decreasing order of total entropy $\left.\frac{d S}{d y}\right|_{y=0}$. For example, for the centrality $0-10 \%$ we pick up the largest $10 \%$ events from entire events. To save computational time, we perform numerical calculation only for our focusing centralities. For the following analysis of experimental data, we prepare 2000 minimum bias events using $\mathrm{T}_{\mathrm{R}}$ ENTo.

In the top panel of Fig. 2 we show pseudorapidity distributions of charged particles in 0-5\%, 10-20\%, 30-40\%, and $50-60 \%$ centralities in the case of $\eta / s=0.08,0.17$, and 0.24 , neglecting the bulk viscosity. The middle panel shows the computational results with finite bulk viscosities and $\eta / s=0.17$. In the bottom panel we include the temperature dependence of $\eta / s$. For all the cases we reproduce centrality dependence of pseudorapidity distributions of charged particles very well (Fig. 22). In TableI we list the values of $N$ and $\sigma_{0}$ which are used in Sec. VB [constant shear viscosity: (a) $\eta / s=0.08$, (b) $\eta / s=0.17$, and (c) $\eta / s=0.24$ ], Sec. VC [finite bulk viscosity: (d) $b=40$ and (e) b=60], and Sec. VD [temperaturedependent shear viscosity: (f) $c_{1}=10, c_{2}=0.7$, (g) $c_{1}=0, c_{2}=0.7$, and $\left.(\mathrm{h}) c_{1}=10, c_{2}=0\right]$. In the case of $(\mathrm{d})$ and $(\mathrm{e})$, we need to choose smaller $\sigma_{0}$ because finite bulk viscosity increases the width of pseudorapidity distributions. We carry out the numerical computation with spatial grid sizes $\Delta x=\Delta y=0.2 \mathrm{fm}, \Delta \eta_{s}=0.3$ and time step $\Delta \tau=0.5 \Delta x \mathrm{fm}$.

TABLE I. The values of normalization $N$ and $\sigma_{0}$ in $\mathrm{T}_{\mathrm{R}}$ ENTo.

\begin{tabular}{|c|ccc|cc|ccc|}
\hline & $(\mathrm{a})$ & $(\mathrm{b})$ & $(\mathrm{c})$ & $(\mathrm{d})$ & $(\mathrm{e})$ & $(\mathrm{f})$ & $(\mathrm{g})$ & $(\mathrm{h})$ \\
\hline$N$ & 116 & 110 & 105 & 94 & 88 & 94 & 98 & 101 \\
$\sigma_{0}$ & 2.9 & 2.9 & 2.9 & 2.7 & 2.7 & 2.9 & 2.9 & 2.9 \\
\hline
\end{tabular}
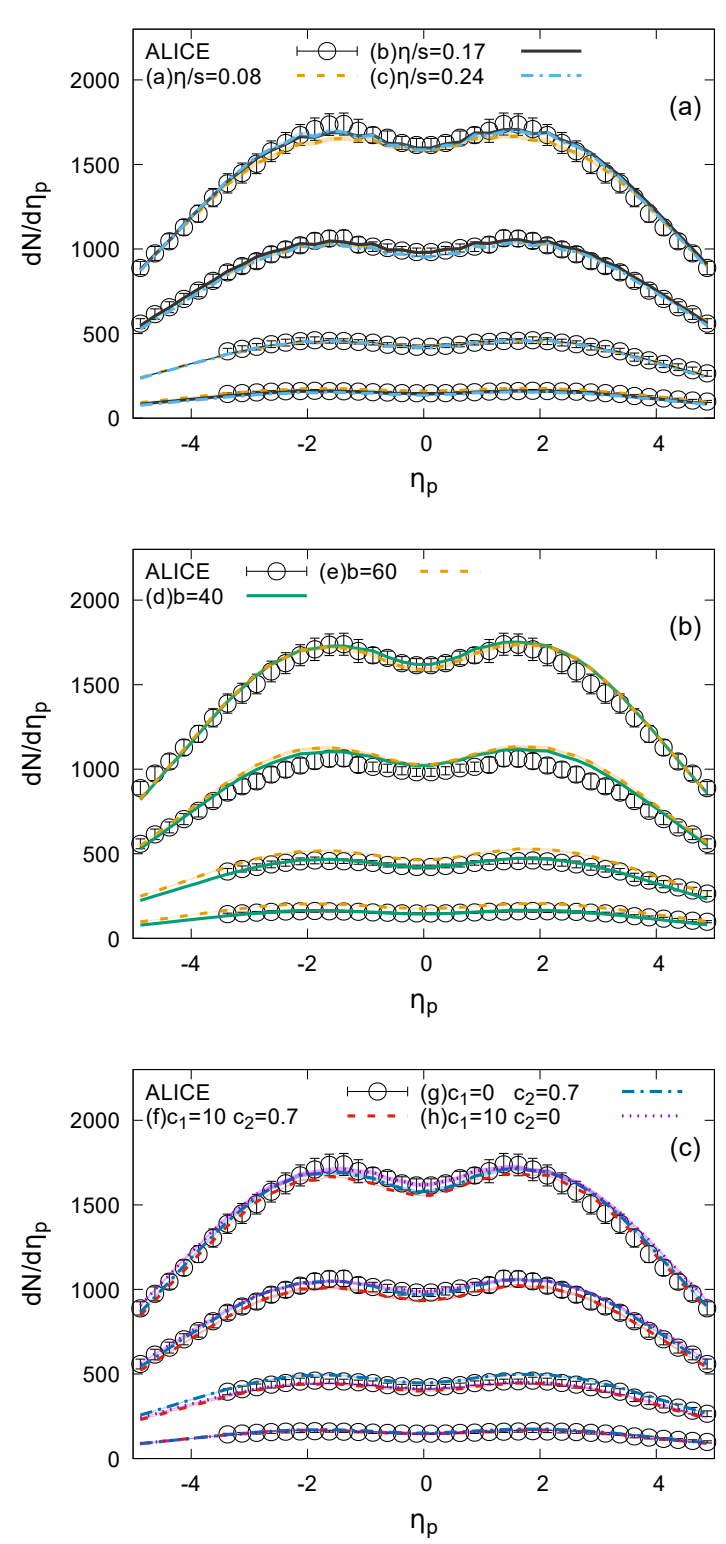

FIG. 2 (color online). The pseudorapidity distributions of charged hadrons in 0-5\%, 10-20\%, 30-40\%, and 50-60\% centralities together with the ALICE data (open circles) 69 . 70. In the top panel, the pseudorapidity distributions were obtained in the case of $\eta / s=0.08,0.17$, and 0.24 at vanishing bulk viscosity (a). The middle panel shows the computational results with shear and bulk viscosities (b). In the bottom panel, temperature dependence of $\eta / s$ is included (c). See the text for details.

\section{B. Constant shear viscosity with vanishing bulk viscosity}

First we extract a suitable value of the shear viscosity $\eta / s$ from comparison with experimental data of $p_{T}$ distributions and collective flows $v_{2}$ and $v_{3}$, neglecting bulk viscosity. In Fig. 3 the $p_{T}$ distributions for $\pi^{+}, K^{+}$, and $p$ are shown, together with the ALICE data [71. The 


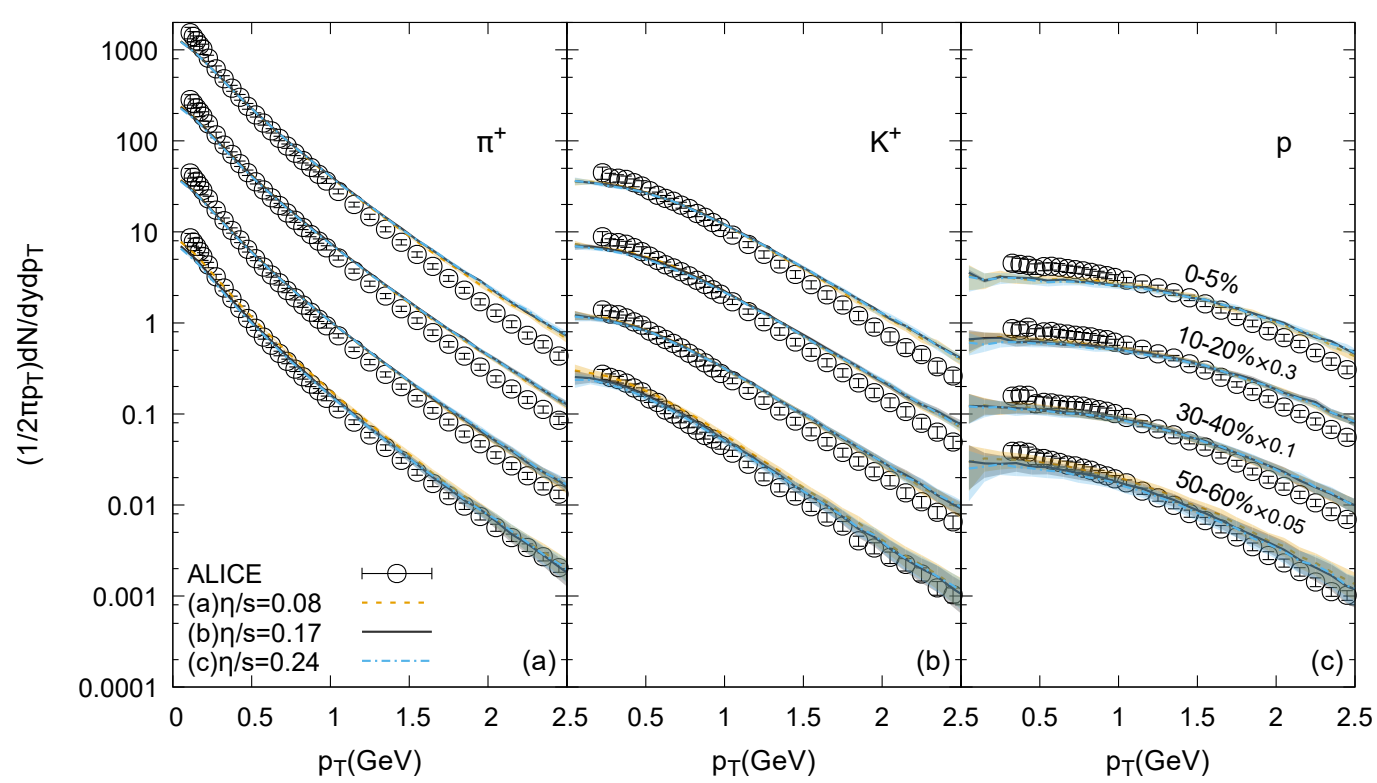

FIG. 3 (color online). The $p_{T}$ distributions for $\pi^{+}(\mathrm{a}), K^{+}(\mathrm{b})$, and $p$ (c) in 0-5\%, 10-20\%, 30-40\%, and 50-60\% centralities, together with the ALICE data (the open circles) [71. The orange dashed line, the black solid line, and the blue dashed-dotted line stand for $\eta / s=0.08,0.17$, and 0.24 , respectively.

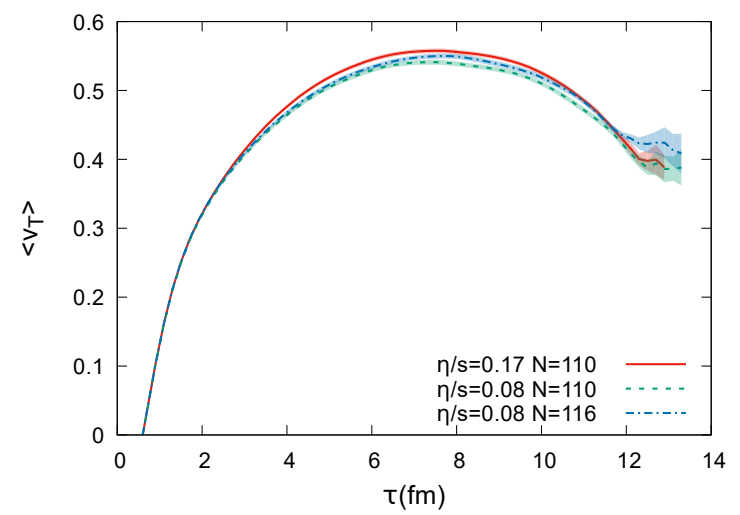

FIG. 4 (color online). The time evolution of the spatial averaged radial flow $v_{T}=\sqrt{v_{x}^{2}+v_{y}^{2}}$ of fluid cells whose temperatures are $T>150 \mathrm{MeV}$ at $\eta_{s}=0$ in $0-5 \%$ centrality. The values are taken from the average over 20 events. The solid line, the dashed line and the dashed-dotted line stand for $\eta / s=0.17$ with $N=110, \eta / s=0.08$ with $N=110$, and $\eta / s=0.08$ with $N=116$, respectively.

differences among calculated results of $p_{T}$ spectra with $\eta / s=0.08,0.17$, and 0.24 are very small, which suggests that $p_{T}$ spectra themselves are not sensitive to the value of $\eta / s$. For all cases we reproduce experimental data reasonably well, though in $0-5 \%$ and $10-20 \%$ centralities we observe a deviation from experimental data above $p_{T} \sim 1.5 \mathrm{GeV}$.

To understand the effect of the shear viscosity on the $p_{T}$ distributions, we show the time evolution of the spatial averaged radial flow $v_{T}=\sqrt{v_{x}^{2}+v_{y}^{2}}$ of fluid cells

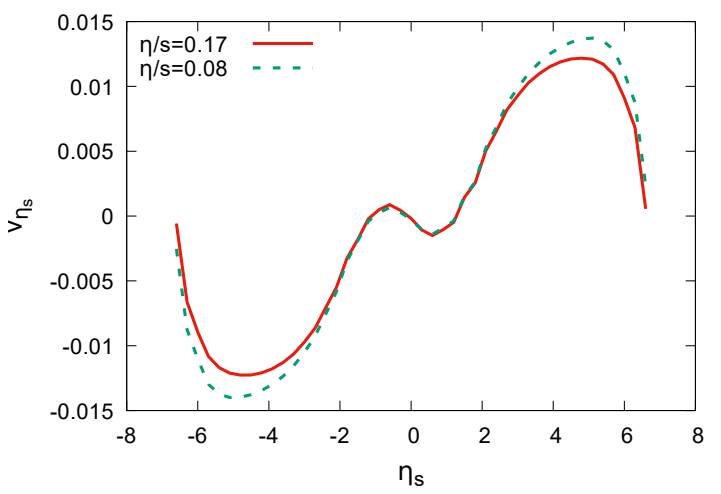

FIG. 5 (color online). The longitudinal flow $v_{\eta_{s}}$ as a function of $\eta_{s}(x=y=0 \mathrm{fm})$ at $\tau=7 \mathrm{fm}$ in $0-5 \%$ centrality. The solid line stands for $\eta / s=0.17$ and the dashed line stands for $\eta / s=$ 0.08. Both of them are computed with the normalization $N=110$ for one event.

whose temperatures are $T>150 \mathrm{MeV}$ at $\eta_{s}=0$ in $0-5 \%$ centrality in Fig. 4. Keeping the normalization $N=110$, we change the value of $\eta / s$ from $\eta / s=0.17$ to $\eta / s=0.08$. With the larger $\eta / s$ the growth of radial flow becomes larger. If we change $N$ from $N=110$ to $N=116$ in the case of $\eta / s=0.08$ to reproduce the rapidity distributions, the difference between $\eta / s=0.17$ and $\eta / s=0.08$ becomes very small. In addition, the average life time of the fluid for $\eta / s=0.17$ is the shortest, since its radial flow is the largest among the three cases.

Figure 5 shows the longitudinal flow as a function of $\eta_{s}$ at $\tau=7 \mathrm{fm}$ in $0-5 \%$ centrality. In the calculation, 

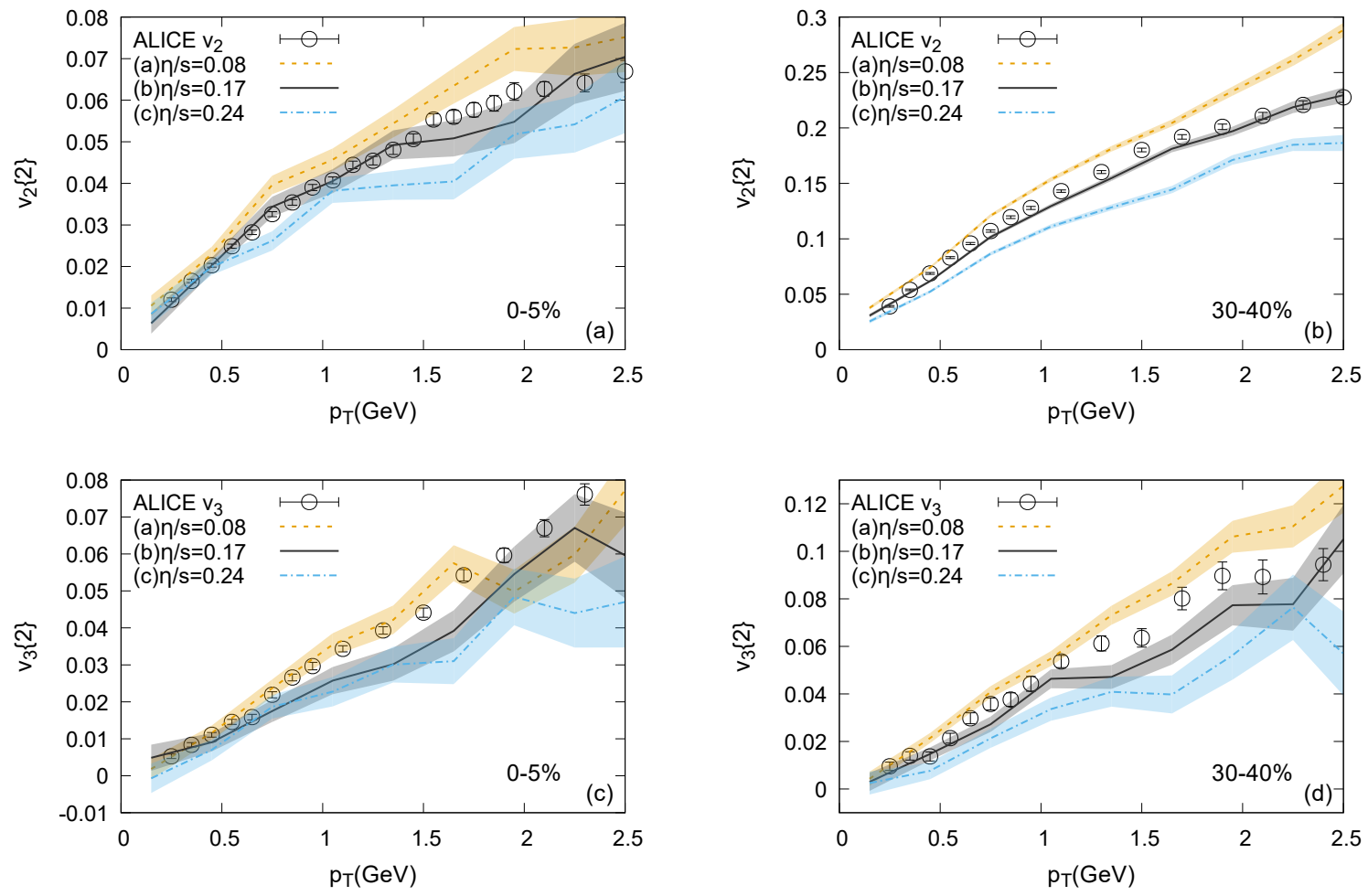

FIG. 6 (color online). The elliptic and triangular flows of charged hadrons as a function of $p_{T}$ in $0-5 \%$ ((a) for $v_{2}$ and (c) for $\left.v_{3}\right)$ and 30-40\% centralities ((b) for $v_{2}$ and (d) for $\left.v_{3}\right)$, together with the ALICE data (the open circles) [72. The orange dashed line, black solid line, and the blue dashed-dotted line stand for $\eta / s=0.08,0.17$, and 0.24 , respectively.

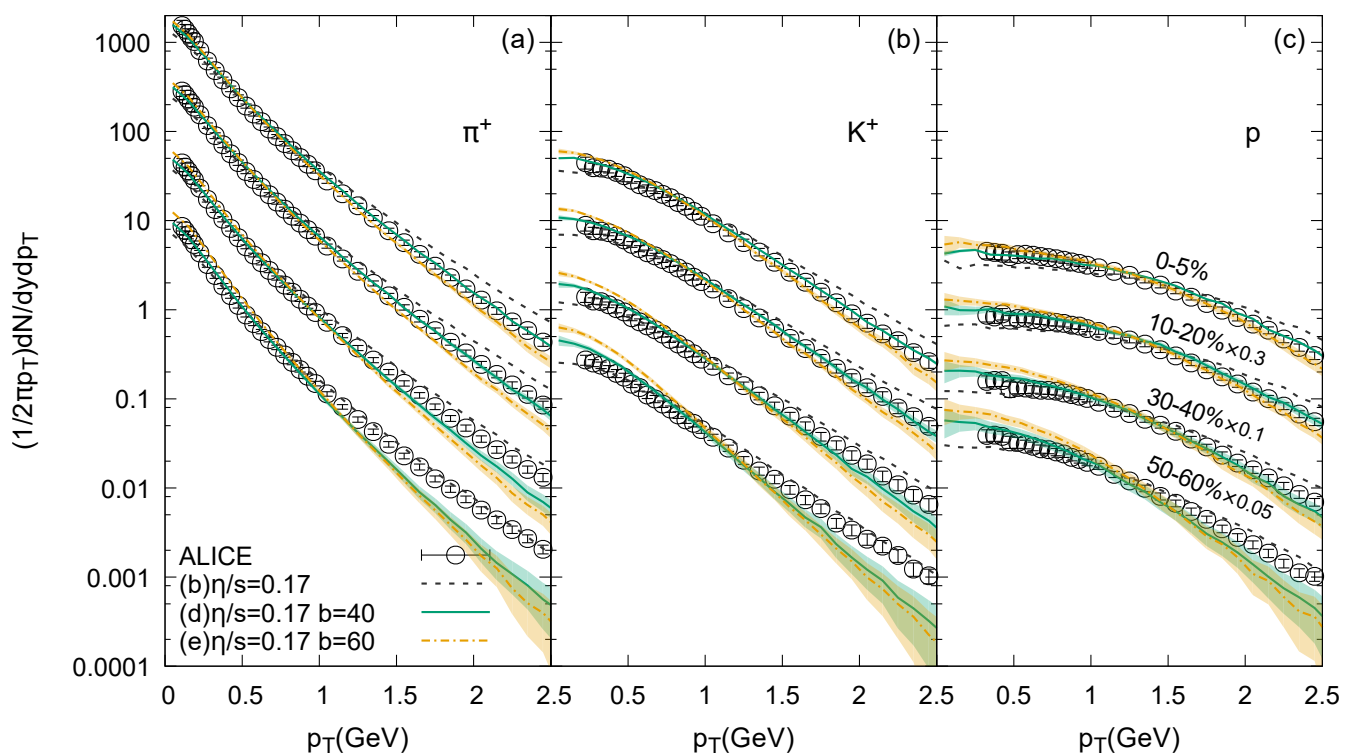

FIG. 7 (color online). The $p_{T}$ distributions for $\pi^{+}(\mathrm{a}), K^{+}$(b), and $p$ (c) in 0-5\%, 10-20\%, 30-40\%, and 50-60\% centralities, together with the ALICE data (the open circles) [71. The black dashed line, the green solid line, and the yellow dashed-dotted line stand for vanishing bulk viscosity, $b=40$, and $b=60$, respectively.

we use the same normalization $N=110$ for $\eta / s=0.08$ and 0.17 . Near midrapidity $\left|\eta_{s}\right|<1$ the sign of $v_{\eta_{s}}$ is changed. The structure comes from the initial condi- tion of pressure distribution of $\mathrm{T}_{\mathrm{R}} \mathrm{ENT}$ in the longitudinal direction: there is a small dent at midrapidity and two bumps beside it. At early times, the initially 


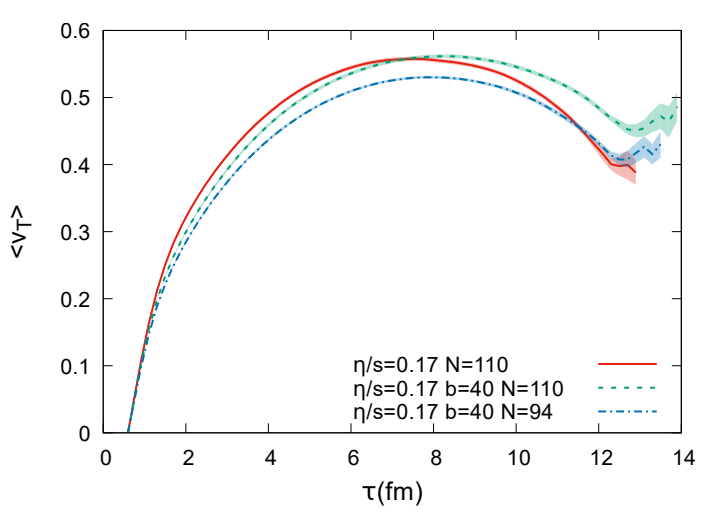

FIG. 8 (color online). The time evolution of the spatial averaged radial flow $v_{T}=\sqrt{v_{x}^{2}+v_{y}^{2}}$ of fluid cells whose temperatures are $T>150 \mathrm{MeV}$ at $\eta_{s}=0$ in $0-5 \%$ centrality. The values are taken from the average over 20 events. The red solid line, the green dashed line, and the blue dasheddotted line stand for $\zeta / s=0$ with $N=110, b=40$ with $N=110$, and $b=40$ with $N=94$, respectively. For all cases we fix the shear viscosity to $\eta / s=0.17$.

produced partons are massless and free-streaming in the $z$ direction, $z / t=p_{z} /|\mathbf{p}|$. Under this assumption, we can estimate $\eta_{s}=\frac{1}{2} \ln \left(\frac{t+z}{t-z}\right) \sim \eta_{p}=\frac{1}{2} \ln \left(\frac{|\boldsymbol{p}|+p_{z}}{|\boldsymbol{p}|-p_{z}}\right)$, which suggests that the dent in pseudo-rapidity distributions also appears in space-time rapidity distributions. Around midrapidity $v_{\eta_{s}}$ becomes smaller than Bjorken's flow, whereas, in $\left|\eta_{s}\right|>2, v_{\eta_{s}}$ is larger than Bjorken's flow due to acceleration from the pressure gradient. Around $\left|\eta_{s}\right| \sim 4$ the difference between two cases becomes large, which means that in the case of larger shear viscosity the growth of longitudinal flow at the early stage of expansion transforms into the larger radial flow. As a result, transverse flow is larger with the larger shear viscosity.

Figure 6 shows the elliptic flow $v_{2}$ and triangular flow $v_{3}$ of charged hadrons as a function of $p_{T}$ in $0-5 \%$ and $30-40 \%$ centralities. We compute the flow harmonics $v_{n}$ from the two-particle cumulant, using the $Q$-cumulant method 73]. The same $p_{T}$ and rapidity cuts as those of the ALICE data 72] are applied. In contrast to the $p_{T}$ distribution Fig. 3. there is clear $\eta / s$ dependence in behavior of collective flows: The larger $\eta / s$ is, the smaller $v_{2}$ and $v_{3}$ are. The existence of shear viscosity suppresses the growth of anisotropy of the flow on the transverse plane. Our result suggests that a suitable $\eta / s$ can be chosen between $\eta / s=0.08$ and $\eta / s=0.17$, which does not contradict the results of Ref. [55].

Here we give a short summary for the constant shear viscosity. The $p_{T}$ spectra of $\pi^{+}, K^{+}$, and $p$ are insensitive to the value of $\eta / s$ and in $0-5 \%$ and $10-20 \%$ centralities our computed $p_{T}$ spectra overestimate above $p_{T}>1.5$ $\mathrm{GeV}$, which suggests that the mean $p_{T}$ is larger. We find the clear $\eta / s$ dependence in $v_{2}$ and $v_{3} ;$ i.e., the larger $\eta / s$ is, the smaller $v_{2}$ and $v_{3}$ of charged hadrons are.
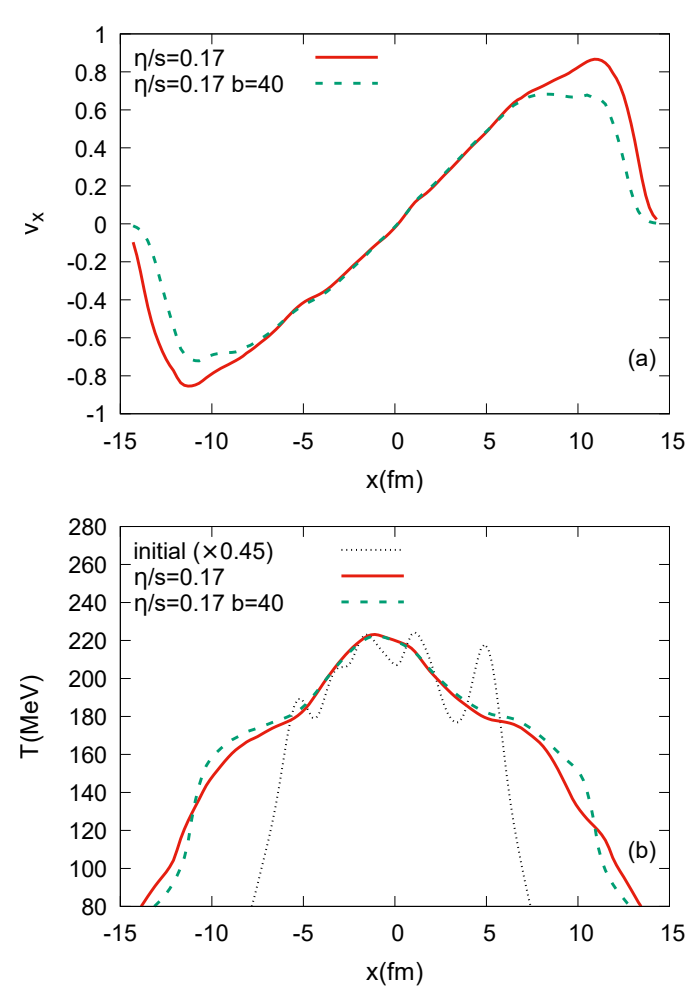

FIG. 9 (color online). The $v_{x}$ (a) and temperature (b) distributions as a function of $x \mathrm{fm}\left(y=0 \mathrm{fm}\right.$ and $\left.\eta_{s}=0\right)$ at $\tau=7$ $\mathrm{fm}$ in $0-5 \%$ centrality. The red solid line stands for $\eta / s=0.17$ with vanishing bulk viscosity and the green dashed line stands for $\eta / s=0.17$ with $b=40$. Both of them are computed with the normalization $N=110$ for one event.

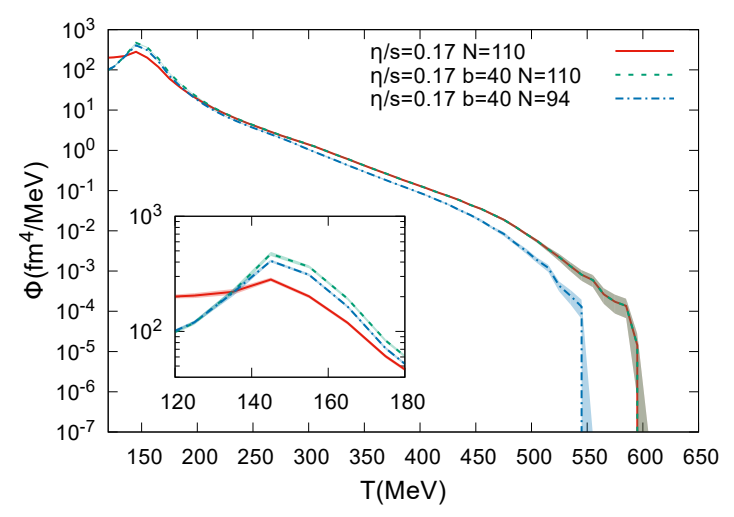

FIG. 10 (color online). The profile functions in $0-5 \%$ centrality. The red solid line, the green dashed line, and the blue dashed-dotted line stand for $\zeta / s=0$ with $N=110, b=40$ with $N=110$, and $b=40$ with $N=94$, respectively. All calculations are done with $\eta / s=0.17$. The values are taken from the average over ten events.

\section{Effect of bulk viscosity}

Next we investigate how the effect of bulk viscosity appears in $p_{T}$ spectra and collective flows $v_{2}$ and $v_{3}$. We introduce the bulk viscosity through Eq. (8), fixing the 

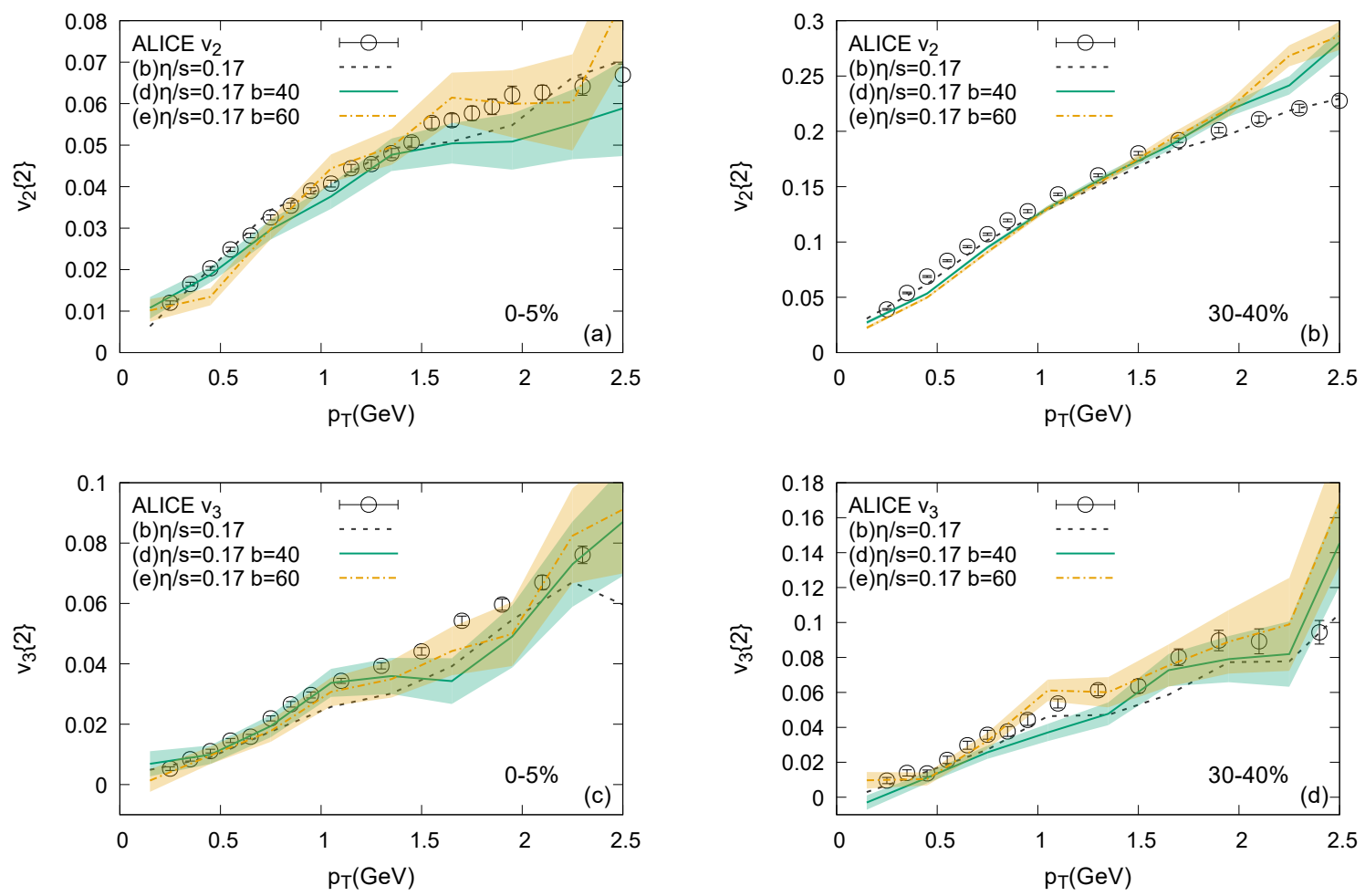

FIG. 11 (color online). The elliptic and triangular flows of charged hadrons as a function of $p_{T}$ in $0-5 \%$ [(a) for $v_{2}$ and (c) for $v_{3}$ ] and 30-40\% [(b) for $v_{2}$ and (d) for $v_{3}$ ] centralities, together with the ALICE data (the open circles) [72]. The black dashed line, the green solid line, and the dashed-dotted line stand for $\eta / s=0.17$ without bulk viscosity, $\eta / s=0.17$ with $b=40$, and $\eta / s=0.17$ with $b=60$, respectively.

value of shear viscosity to $\eta / s=0.17$.

Figure 7 shows the transverse momentum distributions for $\pi^{+}, K^{+}$, and $p$ in $0-5 \%, 10-20 \%, 30-40 \%$, and 50-60 $\%$ centralities. The slopes of $p_{T}$ spectra of $\pi^{+}, K^{+}$, and $p$ are steeper, if the value of bulk viscosity is larger. The growth of the transverse flow becomes small due to the existence of bulk viscosity.

To understand the detailed feature of the behavior of $p_{T}$ spectra, we investigate the time evolution of the transverse flow in Fig. 8. From comparison between the red solid line $(\eta / s=0.17, N=110)$ and the green dashed line $(\eta / s=0.17, b=40, N=110)$ we can see the bulk viscosity effect. The existence of bulk viscosity delays the growth of the transverse flow until around $\tau=7.0$ fm. After $\tau=7.0 \mathrm{fm}$, the $\left\langle v_{T}\right\rangle$ of the green dashed line is larger than that of the red solid line, in turn. On the other hand, if we change the normalization $N$ from $N=110$ to $N=94$ to reproduce the rapidity distributions in Fig. 2, almost all time of expansion $\left\langle v_{T}\right\rangle$ of the blue dashed-dotted line $(\eta / s=0.17, b=40, N=94)$ is smaller than that of the red solid line $(\eta / s=0.17$, $\zeta / s=0)$. Therefore the slope of the $p_{T}$ distribution with larger bulk viscosity becomes steeper than that without bulk viscosity in Fig. 7.

Furthermore we investigate the bulk viscosity effect in the $v_{x}$ and temperature distributions as a function of $x$ $\left(y=0 \mathrm{fm}\right.$ and $\left.\eta_{s}=0\right)$ at $\tau=7 \mathrm{fm}$ in $0-5 \%$ centrality in Fig. 9. In our parametrization of the bulk viscosity Eq. (8), it becomes large below $T<200 \mathrm{MeV}$ (Fig. 1). As a result, around $|x| \sim 10 \mathrm{fm}$ growth of $v_{x}$ is suppressed and diminution of temperature is smaller, in the case of finite bulk viscosity. The fraction of fluid elements whose temperature is around the critical temperature becomes large. On the other hand, around $x \sim 0 \mathrm{fm}$ where the temperature is above $200 \mathrm{MeV}$ the bulk viscosity does not affect $v_{x}$ and temperature distributions.

To understand the bulk viscosity effect in the temperature distributions, we evaluate the profile function [74] in Fig. 10 .

$$
\Phi(T)=\int d^{4} x \delta(T(x)-T)
$$

Here we can see that in the case of finite bulk viscosity the profile functions around the critical temperature are enhanced. The enhancement may affect physical observables such as the photon and lepton-pair production in the medium.

In Fig. 11 we show the elliptic and triangular flows of charged hadrons as a function of $p_{T}$ in $0-5 \%$ and 30$40 \%$ centralities. Here we display calculated results of $v_{2}$ and $v_{3}$ for $b=40$ and 60 . In spite of our limited statistics, we find the following tendency. At low $p_{T}$, the 


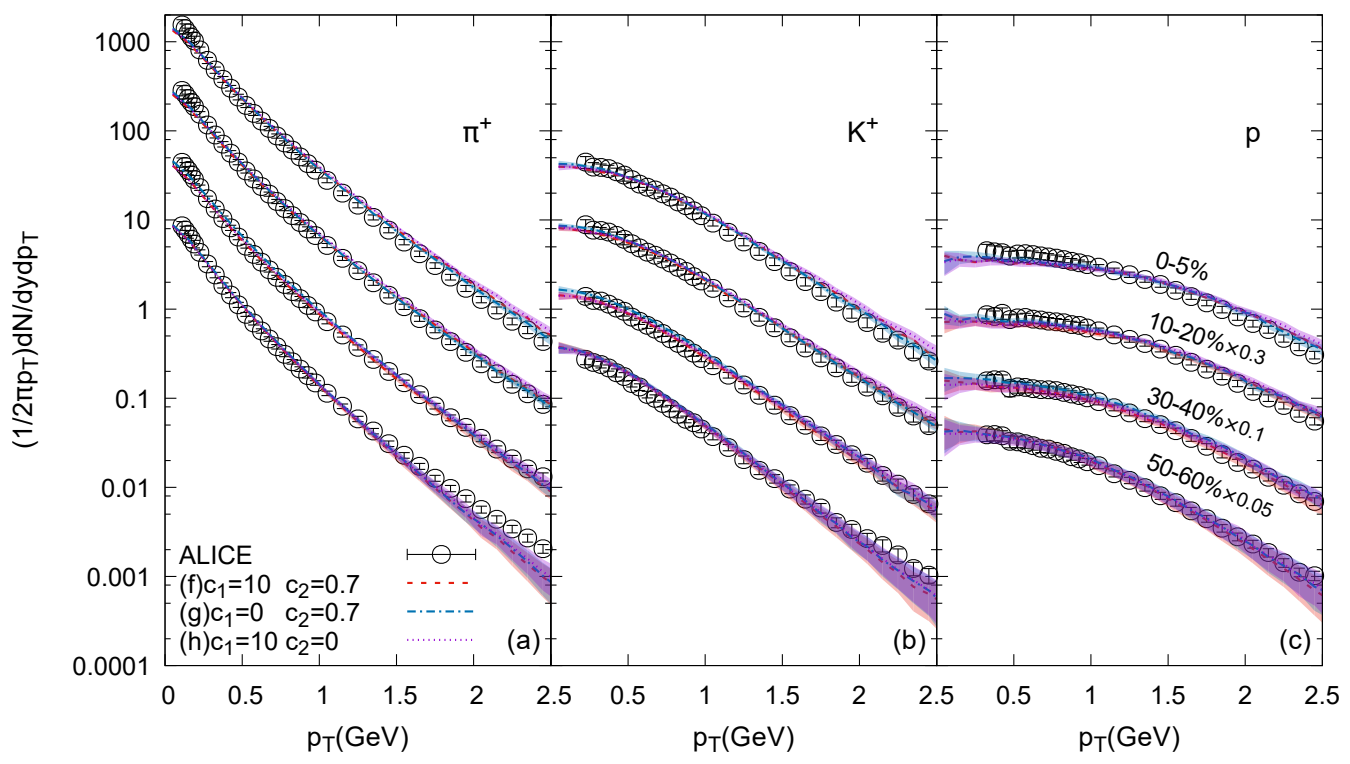

FIG. 12 (color online). The $p_{T}$ distributions for $\pi^{+}$(a), $K^{+}$(b), and $p$ (c) in 0-5\%, 10-20\%, 30-40\%, and 50-60\% centralities together with the ALICE data (the open circles) [71. The red dashed line, the blue dashed-dotted line, and the purple dotted line stand for (f) $c_{1}=10, c_{2}=0.7,(\mathrm{~g}) c_{1}=0, c_{2}=0.7$, and $(\mathrm{h}) c_{1}=10, c_{2}=0.7$, respectively. See the details in the text.

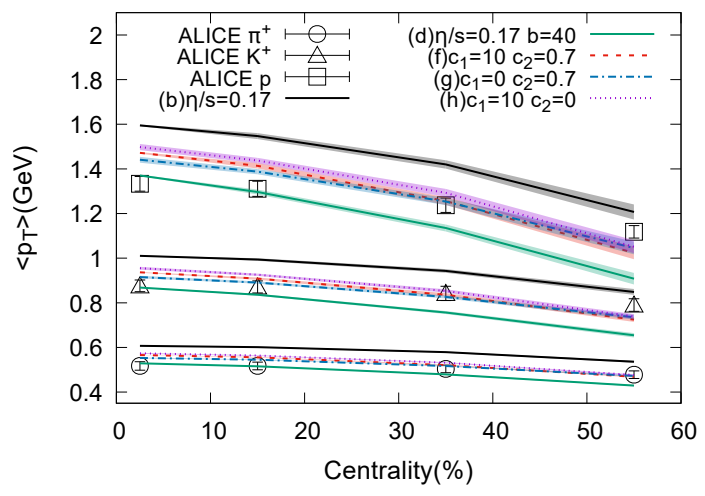

FIG. 13 (color online). The mean $p_{T}$ of $\pi^{+}$(open circles), $K^{+}$(open triangles), and $p$ (open squares) as a function of centrality, together with the ALICE data [1]. We evaluate the mean $p_{T}$ for the whole region of calculated $p_{T}, p_{T}>0$ $\mathrm{GeV}$.

elliptic flow is smaller with larger bulk viscosity. On the other hand, above $p_{T} \sim 2 \mathrm{GeV}$ it is larger with larger bulk viscosity. Because the slope of $p_{T}$ spectra is steeper with larger bulk viscosity (Fig. 7), the anisotropy of flow is smaller (larger) at low (high) $p_{T}$. For the triangular flow, we do not find clear dependence of bulk viscosity in $0-5 \%$ centrality, however, in $30-40 \%$ centrality we observe enhancement of $v_{3}$ with larger bulk viscosity.

For the finite bulk viscosity, we obtain the following results. The slope of $p_{T}$ spectra of $\pi^{+}, K^{+}$, and $p$ becomes steep in the finite bulk viscosity, which suggests a small mean $p_{T}$. The elliptic flow $v_{2}$ becomes small at low $p_{T}$, whereas above $p_{T}>2 \mathrm{GeV}$ it becomes large. The

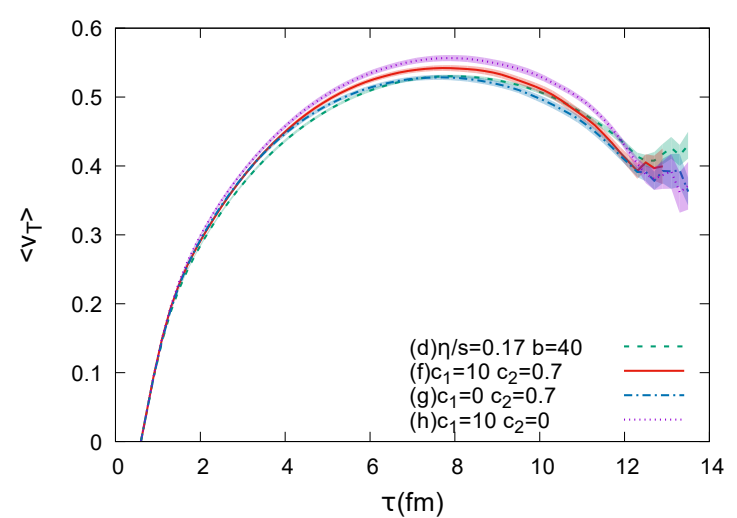

FIG. 14 (color online). The time evolution of the spatial averaged radial flow $v_{T}=\sqrt{v_{x}^{2}+v_{y}^{2}}$ of fluid cells whose temperatures are $T>150 \mathrm{MeV}$ at $\eta_{s}=0$ in $0-5 \%$ centrality, for cases (d), (f), (g) and (h). The values are taken from the average over 20 events.

triangular flow $v_{3}$ is enhanced for the larger bulk viscosity in 30-40\% centrality. Furthermore we find the bulk viscosity effect as the enhancement of the profile function around the critical temperature which may affect physical observables.

\section{Temperature-dependent $\eta / s(T)$ and $\zeta / s(T)$}

We investigate the temperature dependence of shear and bulk viscosities from comparison with the ALICE data. Because the bulk viscous correction to the distribution function is neglected, there should be uncer- 

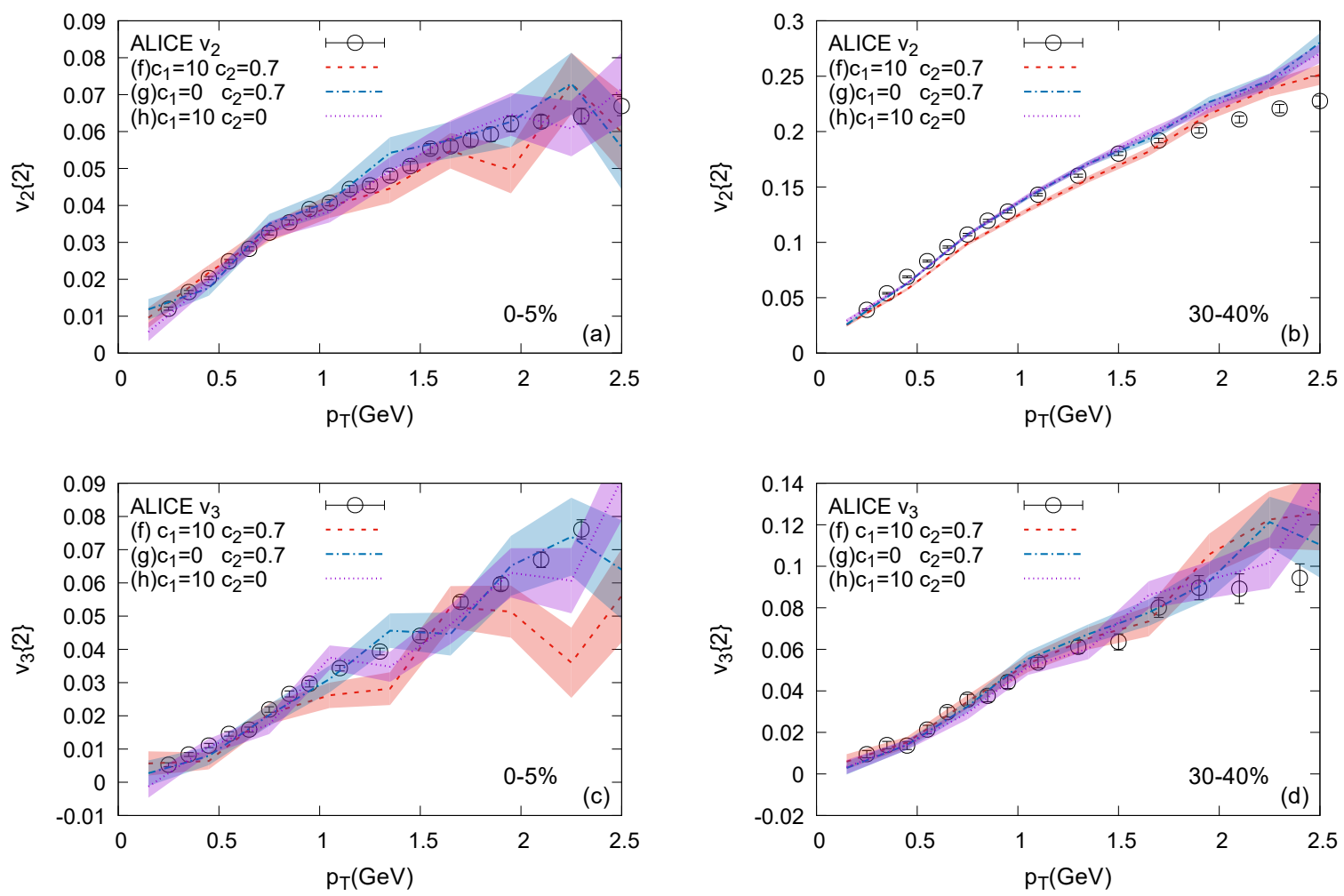

FIG. 15 (color online). The elliptic and triangular flows of charged hadrons as a function of $p_{T}$ in 0-5 \% [(a) for $v_{2}$ and (c) for $\left.v_{3}\right]$ and 30-40\%[(b) for $v_{2}$ and (d) for $\left.v_{3}\right]$ centralities, together with the ALICE data (the open circles) [72]. The red dashed line, the blue dashed, and purple dotted line stand for (f) $c_{1}=10, c_{2}=0.7$, (g) $c_{1}=0, c_{2}=0.7$, and (h) $c_{1}=10, c_{2}=0.7$, respectively.

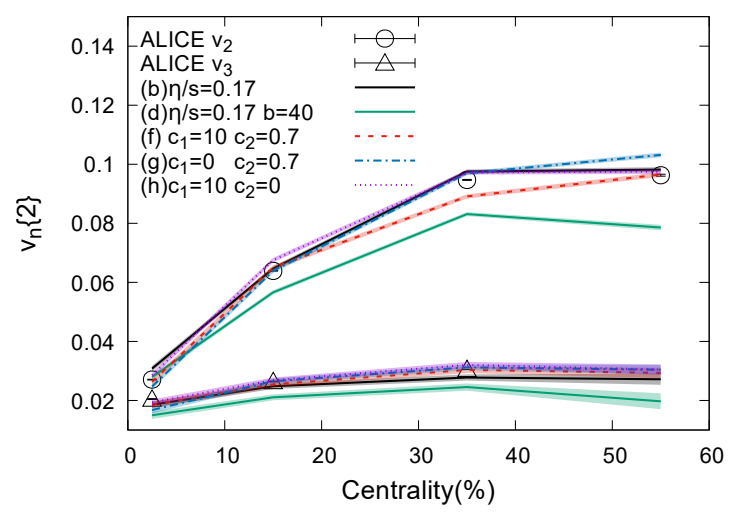

FIG. 16 (color online). The integrated $v_{2}$ and $v_{3}$ of charged hadrons as a function of centrality in the cases of (b) $\eta / s=$ 0.17 (the black solid line), (d) $\eta / s=0.17, b=40$ (the green solid line), (f) $c_{1}=10, c_{2}=0.7$ (the red dashed line), (g) $c_{1}=$ $0, c_{2}=0.7$ (the blue dashed-dotted line), and (h) $c_{1}=10, c_{2}=$ 0 (the purple dotted line). The open circles and squares stand for the $v_{2}$ and $v_{3}$ of the ALICE data, respectively [72].

tainties in determination of the value of $b$ itself. Here we use $b=40$ as one of the possible values for $b$. We shall discuss the consequences of different temperature- dependent $\eta / s(T)$ and $\zeta / s(T)$ models. First in Fig. 12 we show the $p_{T}$ distributions for $\pi^{+}, K^{+}$, and $p$ in $0-5 \%$, 10-20\%, 30-40\%, and 50-60\% centralities, together with the ALICE data. From the $p_{T}$ spectra we can mainly extract the bulk viscosity effect. The $p_{T}$ spectra for (f), $(\mathrm{g})$, and $(\mathrm{h})$ are almost identical, which means that the bulk viscosity effect during hydrodynamic expansion in the three cases is the same. Also, compared with those in Fig. 7, the slope of our computed $p_{T}$ spectra becomes flat and shows better agreement with experimental data. Introduction of the temperature dependence of $\eta / s$ reduces the average value of $\zeta / s$ during hydrodynamic expansion through Eq. (8) (Fig. 1).

In Fig. 13, we show the centrality dependence of mean $p_{T}$ of $\pi^{+}, K^{+}$and $p$, together with the ALICE data [71. All computational results show reasonable agreement with experimental data, except for the case of constant $\eta / s=0.17$ with vanishing bulk viscosity. In the case of (b), our calculated values of $\left\langle p_{T}\right\rangle$ are larger than experimental data, which implies that in our calculation radial flow grows stronger. In particular, a large deviation from experimental data exists in radial flow of protons. Centrality dependence of mean $p_{T}$ of our computational results shows steeper decrease with centrality, compared with experimental data. If bulk viscosity is included, our calculated results of (d), (f), (g), and (h) 

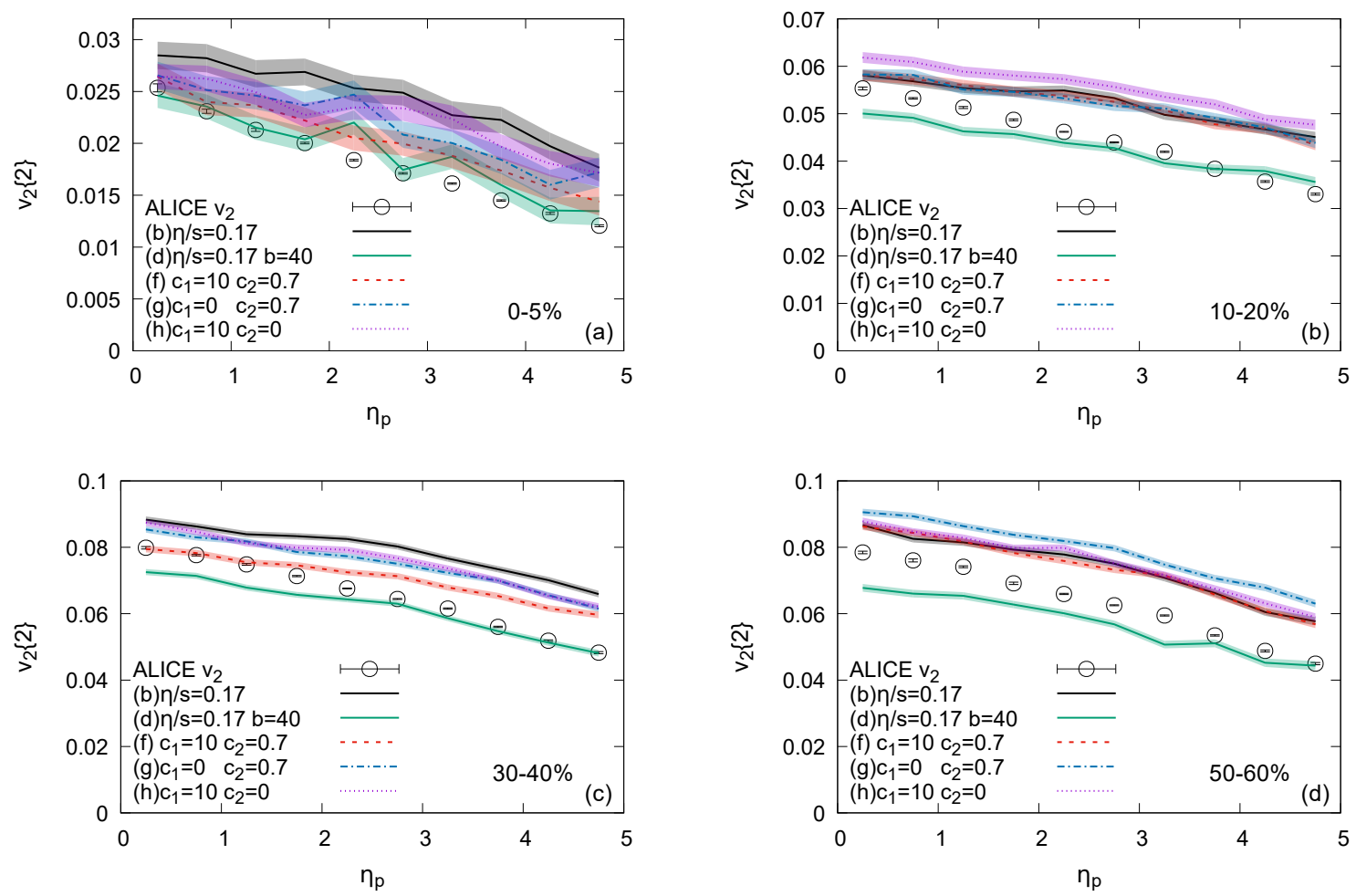

FIG. 17 (color online). Calculated results of integrated $v_{2}$ as a function of pseudorapidity in centralities 0-5 $\%$ (a), $10-20 \%$ (b), 30-40\% (c), and 50-60\% (d), in the cases of (b) $\eta / s=0.17$ (the black solid line), (d) $\eta / s=0.17, b=40$ (the green solid line), (f) $c_{1}=10, c_{2}=0.7$ (the red dashed line), (g) $c_{1}=0, c_{2}=0.7$ (the blue dashed-dotted line), and (h) $c_{1}=10, c_{2}=0($ the purple dotted line), together with the ALICE data (the open circles) 75].

become close to experimental data, because bulk viscosity suppresses the growth of radial flow [32, 33]. Our calculations of (d) show good agreement with experimental data up to centrality 10-20\%; however, they deviate from the experimental data at peripheral collisions. In peripheral collisions the suppression due to bulk viscosity is too strong and the mean $p_{T}$ shows rapid decrease with centrality. We find that the mean $p_{T}$ is not sensitive to the differences of the temperature dependence of $\eta / s$ [cases (f), (g), and (h)], as expected from $p_{T}$ spectra of $\pi^{+}, K^{+}$, and $p$.

We examine the time evolution of the transverse flow in $0-5 \%$ centrality in Fig. 14 . Here we focus on four cases (d), (f), (g) and (h). Until $\tau \sim 8$ fm the order of the magnitude of $\left\langle v_{T}\right\rangle$ is the same as that of mean $p_{T}$ : (h), (f), (g) and (d) in descending order. After $\tau \sim 8 \mathrm{fm}$ $\left\langle v_{T}\right\rangle$ of case (d) becomes larger than that of case (g). It suggests that mean $p_{T}$ is determined mainly by the early stage of the expansion.

Figure 15 shows the elliptic and triangular flows of charged hadrons as a function of $p_{T}$ in $0-5 \%$ and 30$40 \%$ centralities. From collective flows we can investigate both shear and bulk viscosities. The results of $p_{T}$ spectra and mean $p_{T}$ suggest that the cases (f), (g), and (h) have almost the same bulk viscosity effect during hydrodynamic expansion. Therefore we can understand the temperature dependent shear-viscosity effect on the collective flows. In both centralities, the elliptic flow of case (f) is the smallest among three cases. In the case of (f) the average value of shear viscosity over fluid cells is largest, which leads the smallest amplitude of elliptic flow among them. There are small differences between cases $(\mathrm{g})$ and $(\mathrm{h})$ in the elliptic flows. This is because in the current parametrization of $\eta / s(T)$ the average values of shear viscosity are almost the same. Furthermore, compared with Fig. 6 enhancement of $v_{2}$ due to finite bulk viscosity is observed above $p_{T}>2 \mathrm{GeV}$. For the triangular flow we find the same tendency in 0-5\% centrality, in spite of errors. In 30-40\% centrality all (f), (g), and (h) cases show good agreement with the experimental data, which is realized by enhancement of $v_{3}$ due to finite bulk viscosity.

Figure 16 shows centrality dependence of integrated $v_{n}$ $(n=2,3)$. The results of case (b) are consistent with the ALICE data 72. However this agreement is accidentally achieved by larger $\left\langle p_{T}\right\rangle$ and smaller $v_{2}\left(p_{T}\right)$. The integrated $v_{n}$ is affected not only by $v_{n}\left(p_{T}\right)$ but also by $p_{T}$ spectra through integration over $0.2<p_{T}<5 \mathrm{GeV}$. The computed $v_{2}$ for the case (d) is smaller than experimental data except for the central collision, because the mean $p_{T}$ is smaller than the experimental data especially in peripheral collisions. Cases $(\mathrm{f}),(\mathrm{g})$, and $(\mathrm{h})$ 

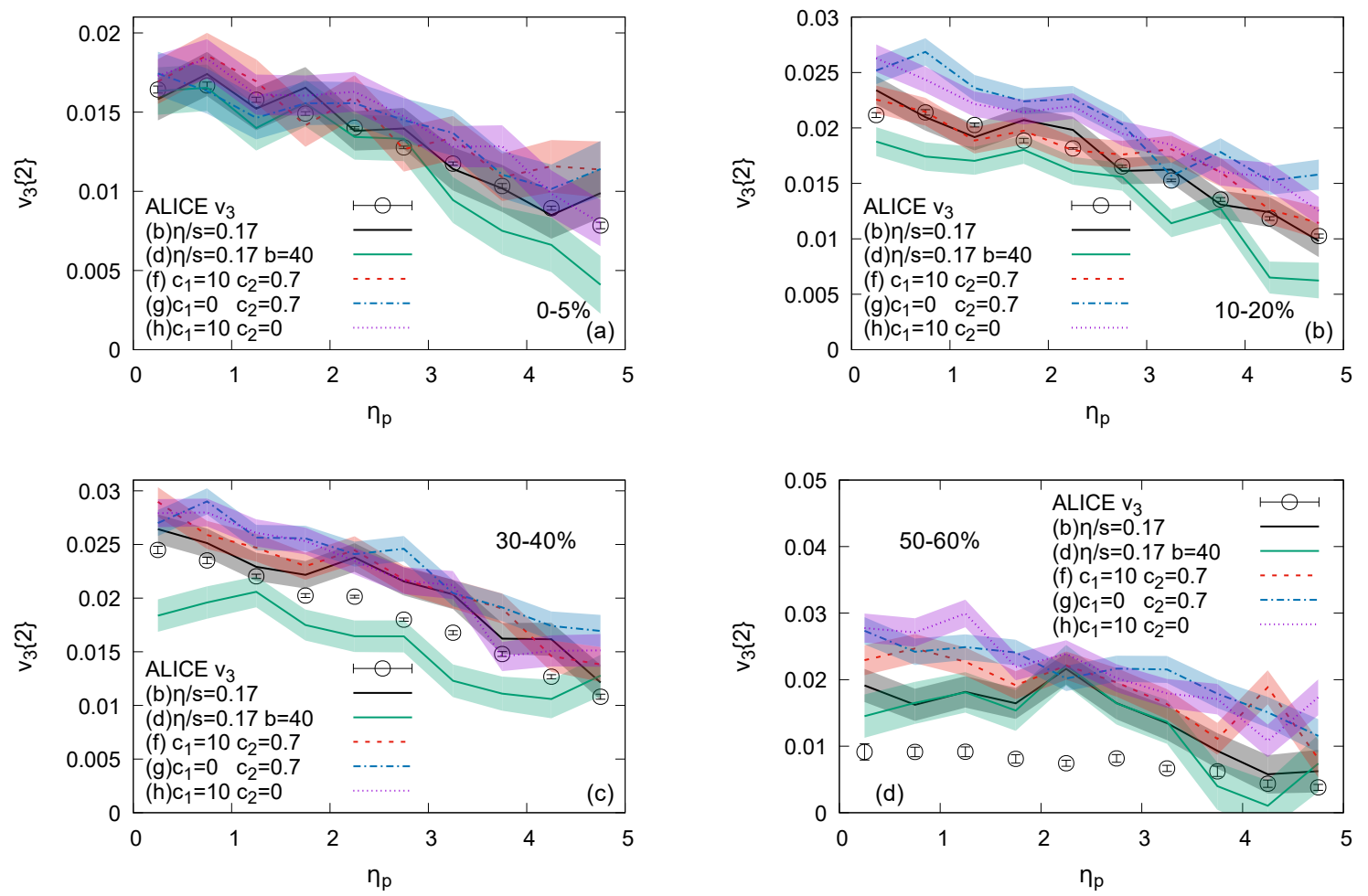

FIG. 18 (color online). Calculated results of integrated $v_{3}$ as a function of pseudorapidity in centralities 0-5 \% (a), 10-20 \% (b), 30-40\% (c), and 50-60\% (d), in the cases of (b) $\eta / s=0.17$ (the black solid line) (d) $\eta / s=0.17, b=40$ (the green solid line), (f) $c_{1}=10, c_{2}=0.7$ (the red dashed line), (g) $c_{1}=0, c_{2}=0.7$ (the blue dashed-dotted line), and (h) $c_{1}=10, c_{2}=0($ the purple dotted line), together with the ALICE data [75].

show good agreement with the experimental data, which is a consequence of consistent with experimental data for $v_{2}\left(p_{T}\right)$ and $p_{T}$ spectra. In $50-60 \%$ centrality $v_{2}$ of case (g) is larger than experimental data, which suggests that in the centrality the shear viscosity of the hadronic phase is important.

We examine the viscosity effects of elliptic flows as a function of pseudorapidity for 0-5\%, 10-20\%, 30-40\%, and 50-60\% centralities in Fig. 17. If we input only the constant $\eta / s$ in the calculations, our results of $v_{2}$ are larger than experimental data and the slope of $v_{2}\left(\eta_{p}\right)$ at peripheral collisions is gentler than that of the ALICE data 75. On the other hand, if we add bulk viscosity [case (d)], the absolute value of $v_{2}\left(\eta_{p}\right)$ becomes small and approaches to the experimental data. Because in the computation of $v_{2}\left(\eta_{p}\right) p_{T}$ integration is performed, the mean $p_{T}$ for cases (b) and (d) affects the amplitude of $v_{2}\left(\eta_{p}\right)$. For cases (f), (g), and (h), their centrality dependence of mean $p_{T}$ as well as behavior of $v_{2}\left(p_{T}\right)$ are almost the same, which means that there should be small differences among them in the behavior of $v_{2}\left(\eta_{p}\right)$. However we find an interesting viscosity effect in the centrality dependence of $v_{2}\left(\eta_{p}\right)$. For the three cases the average bulk viscosity effect during hydrodynamic expansion is almost the same. It means that different behavior of $v_{2}\left(\eta_{p}\right)$ among them originates mainly from different temperature dependence of shear viscosity. In 10-20\% centrality, $v_{2}\left(\eta_{p}\right)$ of case $(\mathrm{h})$ becomes the largest. In the centrality shear viscosity of the QGP phase is dominant. In $30-40 \%$ centrality, $v_{2}\left(\eta_{p}\right)$ of case (f) shows the smallest value, which suggests that shear viscosity of the QGP and hadronic phases important. In 50-60\% centrality $v_{2}\left(\eta_{p}\right)$ of case $(\mathrm{g})$ becomes the largest, which suggests that shear viscosity of the hadronic phase is important [22, 24]. The centrality dependence of $v_{2}\left(\eta_{p}\right)$ reveals the detailed temperature dependence of $\eta / s$ and $\zeta / s$. The deviation between our results and the experimental data becomes large at forward rapidity.

Here we make a comment on behavior of $v_{2}\left(\eta_{p}\right)$ at RHIC, which also shows rapid decrease at forward and backward $\eta_{p}$. It is understood by introduction of temperature dependent shear viscosity in the hadronic phase 24. On the other hand, in our results for the LHC we do not find the clear difference between the slope of $v_{2}\left(\eta_{p}\right)$ of cases (b) and (h), because in our parametrization the average value of shear viscosity during hydrodynamic expansion between the two cases is almost the same. The shape of the $v_{2}\left(\eta_{p}\right)$ is determined not only by the shear viscosity of the hadronic phase but also by the average value of shear viscosity of the fluid. Furthermore since $v_{2}\left(\eta_{p}\right)$ is evaluated by the correlation between particles at midrapidity and those at forward rapidity, decorrelation 

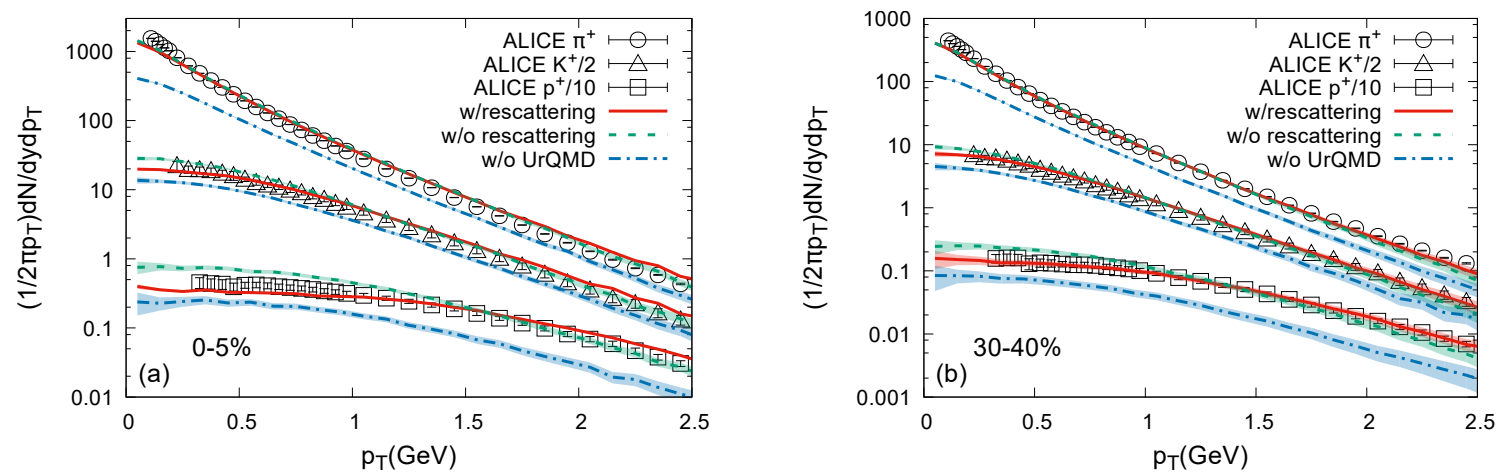

FIG. 19 (color online). The $p_{T}$ distributions for $\pi^{+}, K^{+}$, and $p$ in 0-5\% (a) and 30-40\% (b) centralities, together with the ALICE data (the open circles) [71. The red solid line, the green dashed line, and the blue dashed-dotted line stand for computed $p_{T}$ spectra with rescattering, without rescattering (only resonance decay), and without UrQMD (just from hydrodynamic evolution), respectively.

between them may need to be considered.

In Fig. 18 we show $v_{3}$ of charged hadrons as a function of pseudorapidity in $0-5 \%, 10-20 \%, 30-40 \%$, and $50-60 \%$ centralities. The errors in the triangular flows are larger than those in the elliptic flows. The case (b) shows good agreement with the experimental data; however, this agreement is realized from the combination of large mean $p_{T}$ (Fig. 13) and small $v_{3}\left(p_{T}\right)$ (Fig. 6). The smaller value of $v_{3}\left(\eta_{p}\right)$ of case (d) comes from smaller mean $p_{T}$. Due to the large errors in the triangular flows cases (f), (g), and (h) are not distinguishable. To reach the conclusive results for the temperature-dependence of $\eta / s$ from $v_{3}\left(\eta_{p}\right)$, we need to perform calculations with more statistics. In 50-60\% centrality $v_{3}\left(\eta_{p}\right)$ for all cases are larger than the experimental data.

For a brief summary, the $p_{T}$ spectra are not sensitive to the parametrization $\eta / s(T)$, but their slopes depend on the average value of $\zeta / s(T)$ during the hydrodynamic expansion: they are steeper with larger $\zeta / s$. From the amplitude of mean $p_{T}$, we can extract the bulk viscosity effect. The collective flows $v_{2}$ and $v_{3}$ are affected by both shear and bulk viscosities. The average value of shear viscosity reduces the amplitude of $v_{2}$ and $v_{3}$, whereas the bulk viscosity reduces $v_{2}$ and $v_{3}$ at low $p_{T}$ and enhances them at high $p_{T}$, which is related to the change of $p_{T}$ slope. Our parametrization of (f), (g), and (h) shows the best agreement with the experimental data compared with other cases [(b) and (d)], which implies that the parametrization $\eta / s$ and $\zeta / s$ in $(\mathrm{f}),(\mathrm{g})$, and (h) is one of the most suitable choices. Furthermore we point out that the agreement with the experimental data found in $\left\langle v_{n}\right\rangle$ with constant $\eta / s$ is realized just from the combination of smaller $v_{2}\left(p_{T}\right)$ and larger $\left\langle p_{T}\right\rangle$. We find the effect of the temperature dependence of $\eta / s$ in the centrality dependence of $v_{n}$ : in the central (peripheral) collisions, viscosities of the hadronic (QGP) phase becomes important.

\section{E. Final state interactions}

Finally we investigate the effect of the final state interactions on $p_{T}$ spectra and collective flows $v_{2}$ and $v_{3}$ in detail. Here we focus on case (f) $\left(c_{1}=10, c_{2}=0.7\right.$ and $\eta / s=0.17)$. Figure 19 shows the $p_{T}$ spectra of $\pi^{+}, K^{+}$, and $p$ in 0-5 \% (left panel) and 30-40\% (right panel) centralities, together with the ALICE data [1]. For the $p_{T}$ spectra just from hydrodynamics at the switching temperature $\left(T_{S W}=150 \mathrm{MeV}\right)$, the slopes are almost the same as those of the experimental data; however, their yields are much less than the experimental data. Once we include the resonance decay, the $p_{T}$ spectra move to close to the experimental data. In particular, for $\pi^{+}$we observe a significant decay effect at low $p_{T}$. Furthermore, during final state interactions, particles earn the transverse momentum so that the slope of $p_{T}$ spectra including rescattering becomes flat compared with that without rescattering. We can clearly see the same tendency in $p_{T}$ spectra of protons.

In Fig. 20, we show the values of mean $\left\langle p_{T}\right\rangle$ of $\pi^{+}$, $K^{+}$, and $p$ with rescattering, without rescattering, and without UrQMD in 0-5 \% (left panel) and 30-40 \% (right panel), together with the ALICE data [71]. We also list the values of $\left\langle p_{T}\right\rangle$ in Table II. In the case of $\pi^{+}$, the resonance decay at low $p_{T}$ is so large that the slope of $p_{T}$ is steeper. As a result $\left\langle p_{T}\right\rangle$ with rescattering and without rescattering becomes small, compared to that without UrQMD. On the other hand, for $\left\langle p_{T}\right\rangle$ of $p$ due to the rescattering in the final state interactions the slope of $p_{T}$ spectra becomes flat, which leads to growth of mean $\left\langle p_{T}\right\rangle$.

In Fig. 21, we investigate the effect of resonance decay and final state interactions from the elliptic flow of charged hadrons in 30-40 \% centrality. We find that most of the elliptic flow develops during the hydrodynamic evolution. It indicates that the elliptic flow reflects the features of QGP fluid such as the EoS and transport coefficients without being smeared by the final state interac- 

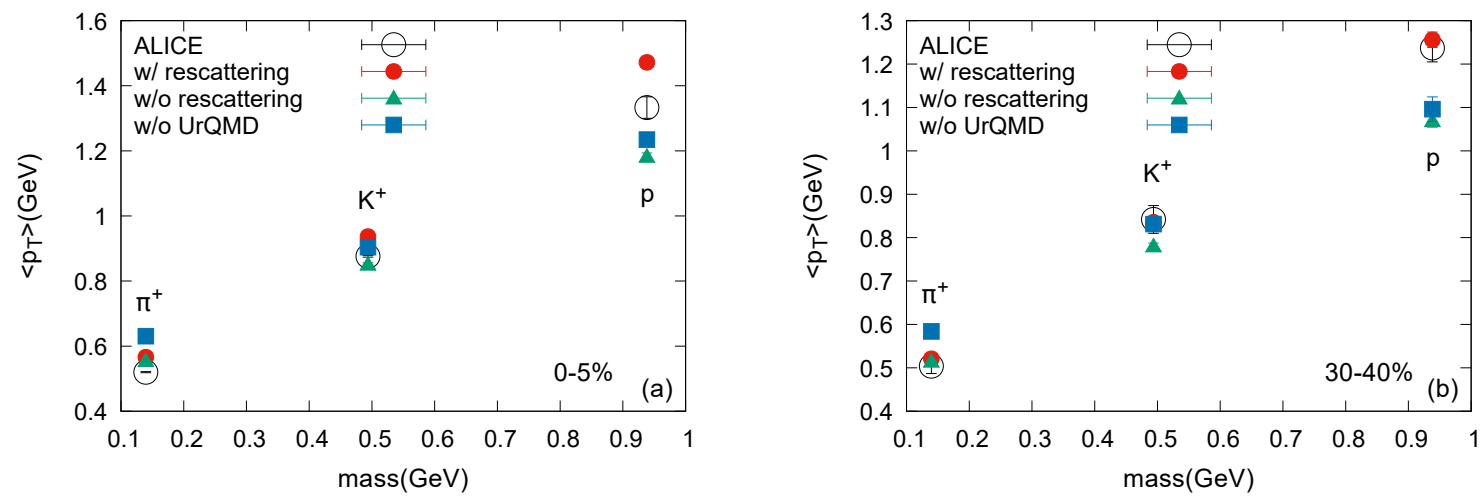

FIG. 20 (color online). The mean $p_{T}$ for $\pi^{+}, K^{+}$, and $p$ with rescattering (the red solid circles), without rescattering (the green solid triangles), and without UrQMD (the blue solid squares) in 0-5\% (a) and 30-40\% centralities (b), together with the ALICE data (the open circles) [71. We evaluate the mean $p_{T}$ in the region of $p_{T}>0 \mathrm{GeV}$.
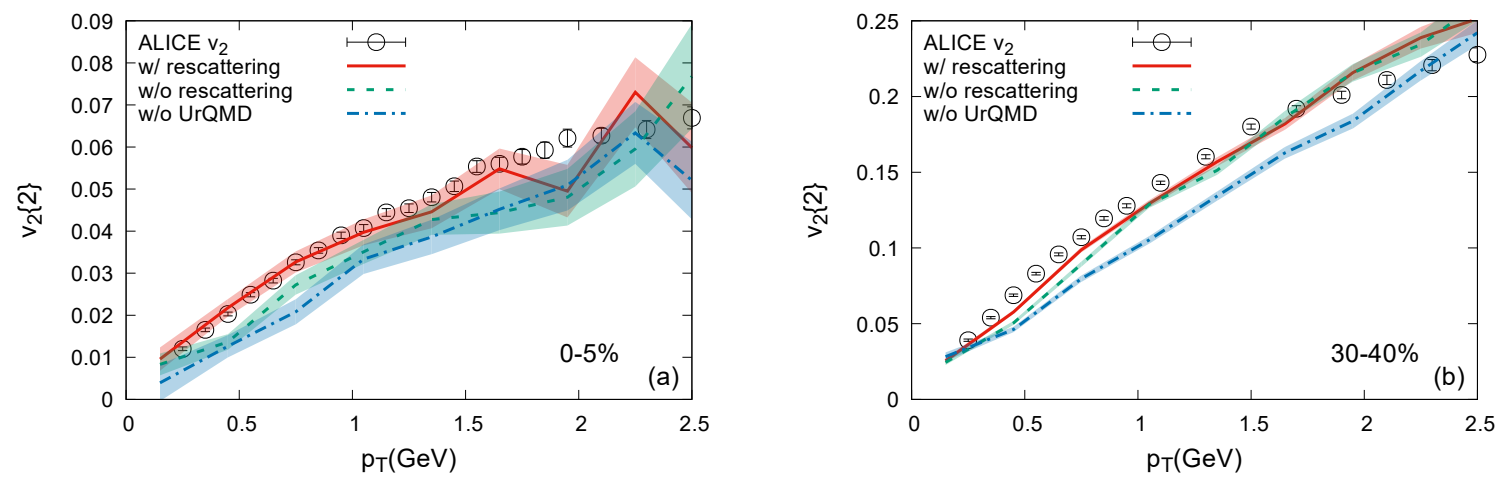

FIG. 21 (color online). The elliptic flow of charged hadrons with rescattering (the red solid line), without rescattering (the blue dashed line), and without UrQMD (the blue dashed-dotted line) as a function of $p_{T}$ in 0-5\% (a) and 30-40\% (b) centralities, together with the ALICE data 72 .

TABLE II. The values of mean $p_{T}$ of $\pi^{+}, K^{+}$, and $p$ with rescattering, without rescattering, and without UrQMD in $0-5 \%$ and $30-40 \%$ centralities.

\begin{tabular}{lllllll}
\hline \hline & & $0-5 \%$ & & $30-40 \%$ & \\
& $\pi^{+}$ & $K^{+}$ & $p$ & $\pi^{+}$ & $K^{+}$ & $p$ \\
\hline w/ rescattering & $0.560 \pm 0.002$ & $0.929 \pm 0.005$ & $1.462 \pm 0.013$ & $0.502 \pm 0.002$ & $0.800 \pm 0.007$ & $1.225 \pm 0.016$ \\
w/o rescattering & $0.546 \pm 0.002$ & $0.837 \pm 0.005$ & $1.168 \pm 0.010$ & $0.493 \pm 0.002$ & $0.749 \pm 0.006$ & $1.031 \pm 0.013$ \\
w/o UrQMD & $0.617 \pm 0.001$ & $0.888 \pm 0.002$ & $1.208 \pm 0.005$ & $0.553 \pm 0.001$ & $0.794 \pm 0.003$ & $1.070 \pm 0.008$ \\
experimental data & $0.517 \pm 0.019$ & $0.876 \pm 0.026$ & $1.333 \pm 0.033$ & $0.504 \pm 0.017$ & $0.842 \pm 0.032$ & $1.237 \pm 0.032$ \\
\hline
\end{tabular}

tions. If the decay effect is included, the elliptic flow of charged hadrons becomes large, close to the experimental data at low $p_{T}$. Particles through decay process tend to be produced in the same direction as that of their parent particles [76, which enhances the amplitude of the elliptic flow 77. On the other hand, we only find small effect from the rescattering on $v_{2}$, comparing $v_{2}$ with rescattering (the red solid line) and that without rescattering (the green dashed line). The change is small; however, elliptic flow with rescattering shows the best agreement with the experimental data.

Finally, in Fig. 22 we compare the elliptic flows of charged hadrons as a function of pseudorapidity with rescattering, without rescattering, and without UrQMD in 0-5\% and 30-40\% centralities. Because $\pi$ is dominant over the produced charged hadrons, the difference among them is explained by focusing $\pi$ in the final state interactions. Interestingly, the elliptic flow without UrQMD shows the largest values, though in Fig. 21 $v_{2}\left(p_{T}\right)$ without UrQMD is the smallest in the whole $p_{T}$ region. From Fig. 20 the value of mean $p_{T}$ without UrQMD of $\pi$ is the largest, which leads to the largest $v_{2}\left(\eta_{p}\right)$ through the $p_{T}$ integral. In other words, mean $p_{T}$ becomes smaller by resonance decay so that $v_{2}\left(\eta_{p}\right)$ without rescattering becomes smaller than that without UrQMD. In addition, the resonance decay only changes the magnitude 

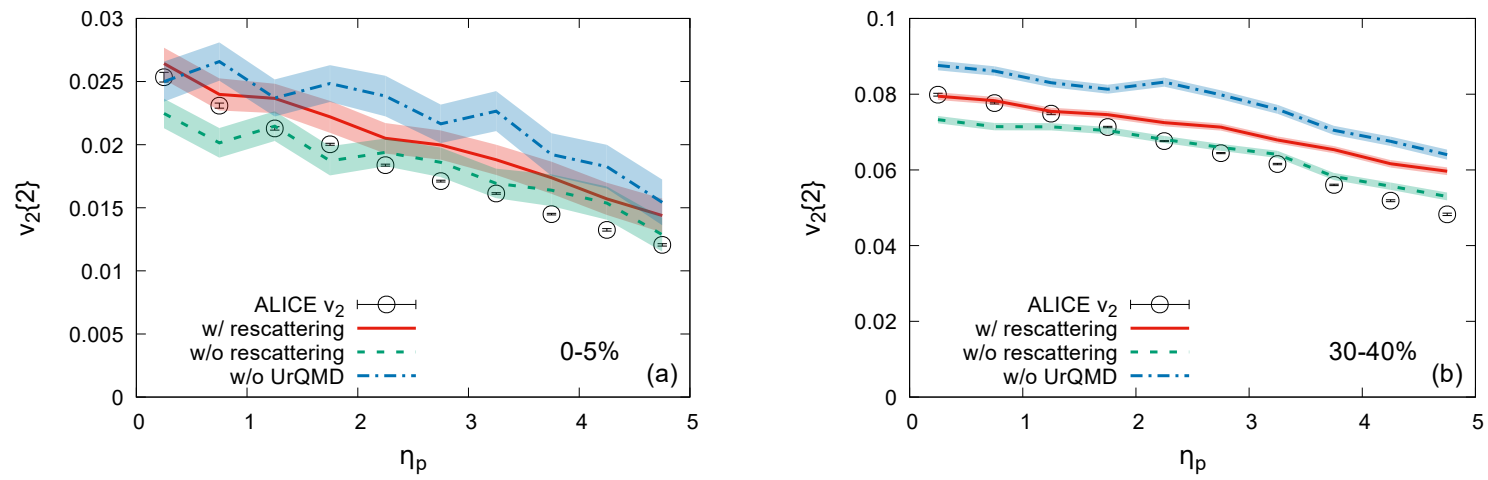

FIG. 22 (color online). The integrated $v_{2}$ as a function of pseudorapidity in centralities 0-5\% (a) and 30-40\% (b), together with the ALICE data [75]. The red solid line stands for $v_{2}$ with rescattering, the green dashed line stands for $v_{2}$ without rescattering, and the blue dashed-dotted line stands for $v_{2}$ without UrQMD.

of $v_{2}\left(\eta_{p}\right)$, but it does not change the $\eta_{p}$ dependence of $v_{2}$. If we include the rescattering in the $v_{2}$, the value of it becomes slightly larger than that without rescattering. This is also understood by the behavior of mean $p_{T}$ of $\pi$ in Fig. 20.

\section{SUMMARY}

We have investigated the temperature dependence of shear and bulk viscosities from comparison with ALICE data: single particle spectra and collective flows at $\mathrm{Pb}+\mathrm{Pb} \sqrt{s_{\mathrm{NN}}}=2.76$ collisions.

First we studied the constant shear viscosity by comparison between our calculated results and the ALICE data. The $p_{T}$ spectra of $\pi^{+}, K^{+}$, and $p$ are insensitive to the value of $\eta / s$, and in $0-5 \%$ and $10-20 \%$ centralities our computed $p_{T}$ spectra overestimate above $p_{T}>1.5$ $\mathrm{GeV}$. We find the clear $\eta / s$ dependence in $v_{2}$ and $v_{3}$; i.e., the larger $\eta / s$ is, the smaller $v_{2}$ and $v_{3}$ of charged hadrons are.

For the finite bulk viscosity, we obtain the following results. The slope of $p_{T}$ spectra of $\pi^{+}, K^{+}$, and $p$ becomes steep in the finite bulk viscosity, which suggests a small mean $p_{T}$. The elliptic flow $v_{2}$ becomes small at low $p_{T}$, whereas above $p_{T}>2 \mathrm{GeV}$ it becomes large. The triangular flow $v_{3}$ is enhanced for the larger bulk viscosity in 30-40\% centrality. Furthermore we find the bulk viscosity effect as the enhancement of the profile function around the critical temperature which may affect physical observables.

Furthermore we have discussed consequences of the temperature dependence of $\eta / s$ and $\zeta / s$. In the parametrization of $\eta / s(T)$ and $\zeta / s(T), p_{T}$ spectra are not sensitive to the parametrization $\eta / s$, but their slopes depend on the average value of $\zeta / s$ during the hydrodynamic expansion. They are steeper with larger $\zeta / s$. From the amplitude of mean $p_{T}$, we can extract the bulkviscosity effect. The collective flows $v_{2}$ and $v_{3}$ are affected by both shear and bulk viscosities. The shear viscosity reduces the amplitude of $v_{2}$ and $v_{3}$. On the other hand, the bulk viscosity reduces $v_{2}$ and $v_{3}$ at low $p_{T}$ and enhances them at high $p_{T}$, which is related to the change of $p_{T}$ slope. Our parametrization of (f), (g), and (h) shows the best agreement with the experimental data compared with other cases $[(\mathrm{b})$ and $(\mathrm{d})]$, which implies that the parametrization $\eta / s$ and $\zeta / s$ in (f), (g), and (h) is one of the most suitable choices. Furthermore we point out that the agreement with the experimental data found in $\left\langle v_{n}\right\rangle$ with constant $\eta / s$ is realized just from the combination of smaller $v_{2}\left(p_{T}\right)$ and larger $\left\langle p_{T}\right\rangle$. We find the effect of the temperature dependence of $\eta / s$ in the centrality dependence of $v_{n}$. In the central (peripheral) collisions, viscosities of the hadronic (QGP) phase becomes important.

Finally we have investigated the effect of the final state interactions. The resonance decays increase yields and the slope of $p_{T}$ spectra becomes flat during final state interactions. Besides, the mean $p_{T}$ of $\pi^{+}$becomes small, whereas that of $p$ becomes large. For elliptic flow as a function of $p_{T}$, most of the elliptic flow develops during the hydrodynamic evolution, though the elliptic flow still continues to grow a little through resonance decays. It indicates that the elliptic flow reflects the feature of QGP fluid such as the EoS and transport coefficients. In $v_{2}\left(\eta_{p}\right)$, through integration of $p_{T}, v_{2}\left(\eta_{p}\right)$ without UrQMD shows the largest value and the resonance decay only changes the magnitude of $v_{2}\left(\eta_{p}\right)$. From the comprehensive analyses of centrality dependence of single particle spectra and collective flow, we can extract the detailed information of the QGP bulk property, without the information being smeared by the final state interactions.

\section{ACKNOWLEDGMENTS}

We would like to thank Marcus Bluhm for providing the code of the lattice QCD-based equations of state as a function of pressure at vanishing net-baryon density. We 
acknowledge valuable discussions on construction of the hybrid model with Jonah E. Bernhard, Weiyao Ke, and J. Scott Moreland. We are grateful for the hospitality of the members of Duke University. Finally we would like to thank Steffen Bass and Berndt Mueller for encouraging us to pursue this work. The work of C.N. is supported by the JSPS KAKENHI Grant-in-Aid for Scientific Research (S) Grant No. JP26220707 and the JSPS KAKENHI Grant-in-Aid for Scientific Research (C) Grant No. JP17K05438.
[1] T.-D. Lee, Nucl. Phys. A750, 1 (2005).

[2] S. Borsanyi, G. Endrodi, Z. Fodor, A. Jakovac, S. D. Katz, S. Krieg, C. Ratti, and K. K. Szabo, JHEP 11, 077 (2010), arXiv:1007.2580 [hep-lat]

[3] A. Bazavov et al., Phys. Rev. D85, 054503 (2012). arXiv:1111.1710 [hep-lat]

[4] M. Prakash, M. Prakash, R. Venugopalan, and G. Welke, Phys. Rept. 227, 321 (1993)

[5] P. B. Arnold, G. D. Moore, and L. G. Yaffe, JHEP 05, 051 (2003), arXiv:hep-ph/0302165 [hep-ph].

[6] P. Kovtun, D. T. Son, and A. O. Starinets, Phys. Rev. Lett. 94, 111601 (2005), arXiv:hep-th/0405231 [hep-th],

[7] L. P. Csernai, J. Kapusta, and L. D. McLerran, Phys. Rev. Lett. 97, 152303 (2006), arXiv:nucl-th/0604032 [nucl-th]

[8] D. Fernandez-Fraile and A. Gomez Nicola, Phys. Rev. Lett. 102, 121601 (2009), arXiv:0809.4663 [hep-ph],

[9] E. Lu and G. D. Moore, Phys. Rev. C83, 044901 (2011), arXiv:1102.0017 [hep-ph]

[10] F. Karsch, D. Kharzeev, and K. Tuchin, Phys. Lett. B663, 217 (2008), arXiv:0711.0914 [hep-ph]

[11] J.-W. Chen, J. Deng, H. Dong, and Q. Wang, Phys. Rev. C87, 024910 (2013), arXiv:1107.0522 [hep-ph]

[12] P. Romatschke and U. Romatschke, Phys. Rev. Lett. 99, 172301 (2007), arXiv:0706.1522 [nucl-th]

[13] M. Luzum and P. Romatschke, Phys. Rev. C78, 034915 (2008), [Erratum: Phys. Rev.C79,039903(2009)], arXiv:0804.4015 [nucl-th],

[14] K. Dusling, G. D. Moore, and D. Teaney, Phys. Rev. C81, 034907 (2010), arXiv:0909.0754 [nucl-th]

[15] C. Shen, U. Heinz, P. Huovinen, and H. Song, Phys. Rev. C82, 054904 (2010), arXiv:1010.1856 [nucl-th]

[16] H. Song, S. A. Bass, U. Heinz, T. Hirano, and C. Shen, Phys. Rev. Lett. 106, 192301 (2011), [Erratum: Phys. Rev. Lett.109,139904(2012)], arXiv:1011.2783 [nucl-th]

[17] C. Gale, S. Jeon, B. Schenke, P. Tribedy, and R. Venugopalan, Phys. Rev. Lett. 110, 012302 (2013), arXiv:1209.6330 [nucl-th].

[18] H.-j. Xu, Z. Li, and H. Song, Phys. Rev. C93, 064905 (2016), arXiv:1602.02029 [nucl-th].

[19] R. D. Weller and P. Romatschke, Phys. Lett. B774, 351 (2017), arXiv:1701.07145 [nucl-th]

[20] H. Niemi, G. S. Denicol, P. Huovinen, E. Molnar, and D. H. Rischke, Phys. Rev. Lett. 106, 212302 (2011), arXiv:1101.2442 [nucl-th],

[21] H. Niemi, G. S. Denicol, P. Huovinen, E. Molnar, and D. H. Rischke, Phys. Rev. C86, 014909 (2012), arXiv:1203.2452 [nucl-th].

[22] E. Molnar, H. Holopainen, P. Huovinen, and H. Niemi, Phys. Rev. C90, 044904 (2014), arXiv:1407.8152 [nuclth],

[23] H. Niemi, K. J. Eskola, and R. Paatelainen, Phys. Rev. C93, 024907 (2016), arXiv:1505.02677 [hep-ph]

[24] G. Denicol, A. Monnai, and B. Schenke, Phys. Rev. Lett.
116, 212301 (2016), arXiv:1512.01538 [nucl-th]

[25] G. S. Denicol, T. Kodama, T. Koide, and P. Mota, Phys. Rev. C80, 064901 (2009), arXiv:0903.3595 [hep-ph].

[26] A. Monnai and T. Hirano, Phys. Rev. C80, 054906 (2009), arXiv:0903.4436 [nucl-th].

[27] H. Song and U. W. Heinz, Phys. Rev. C81, 024905 (2010), arXiv:0909.1549 [nucl-th]

[28] P. Bozek, Phys. Rev. C81, 034909 (2010), arXiv:0911.2397 [nucl-th]

[29] P. Bozek and I. Wyskiel-Piekarska, Phys. Rev. C85, 064915 (2012), arXiv:1203.6513 [nucl-th]

[30] J. Noronha-Hostler, G. S. Denicol, J. Noronha, R. P. G. Andrade, and F. Grassi, Phys. Rev. C88, 044916 (2013), arXiv:1305.1981 [nucl-th]

[31] J. Noronha-Hostler, J. Noronha, and F. Grassi, Phys. Rev. C90, 034907 (2014), arXiv:1406.3333 [nucl-th].

[32] J.-B. Rose, J.-F. Paquet, G. S. Denicol, M. Luzum, B. Schenke, S. Jeon, and C. Gale, Proceedings, 24th International Conference on Ultra-Relativistic NucleusNucleus Collisions (Quark Matter 2014): Darmstadt, Germany, May 19-24, 2014, Nucl. Phys. A931, 926 (2014), arXiv:1408.0024 [nucl-th]

[33] S. Ryu, J. F. Paquet, C. Shen, G. S. Denicol, B. Schenke, S. Jeon, and C. Gale, Phys. Rev. Lett. 115, 132301 (2015), arXiv:1502.01675 [nucl-th].

[34] J. E. Bernhard, J. S. Moreland, S. A. Bass, J. Liu, and U. Heinz, Phys. Rev. C94, 024907 (2016), arXiv:1605.03954 [nucl-th]

[35] S. Ryu, J.-F. Paquet, C. Shen, G. Denicol, B. Schenke, S. Jeon, and C. Gale, Phys. Rev. C97, 034910 (2018), arXiv:1704.04216 [nucl-th]

[36] K. Okamoto and C. Nonaka, Eur. Phys. J. C77, 383 (2017), arXiv:1703.01473 [nucl-th].

[37] M. Takamoto and S.-i. Inutsuka, J. Comput. Phys. 230, 7002 (2011), arXiv:1106.1732 [astro-ph.HE]

[38] K. Okamoto, Y. Akamatsu, and C. Nonaka, Eur. Phys. J. C76, 579 (2016), arXiv:1607.03630 [nucl-th]

[39] Y. Akamatsu, S.-i. Inutsuka, C. Nonaka, and M. Takamoto, J. Comput. Phys. 256, 34 (2014), arXiv:1302.1665 [nucl-th]

[40] L. Landau and E. Lifshitz, Fluid Mechanics, 2nd ed., Course of Theoretical Physic, Vol. 6 (ButterworthHeinemann, Oxford, 1987).

[41] W. A. Hiscock and L. Lindblom, Annals Phys. 151, 466 (1983)

[42] W. A. Hiscock and L. Lindblom, Phys. Rev. D31, 725 (1985)

[43] S. Pu, T. Koide, and D. H. Rischke, Phys. Rev. D81, 114039 (2010), arXiv:0907.3906 [hep-ph].

[44] J. Stewart, Proc. R. Soc. LondonPhys. A357, 59 (1977).

[45] W. Israel and J. M. Stewart, Annals Phys. 118, 341 (1979).

[46] P. Huovinen and D. Molnar, Phys. Rev. C79, 014906 (2009), arXiv:0808.0953 [nucl-th] 
[47] I. Bouras, E. Molnar, H. Niemi, Z. Xu, A. El, O. Fochler, C. Greiner, and D. H. Rischke, Phys. Rev. C82, 024910 (2010), arXiv:1006.0387 [hep-ph].

[48] M. Takamoto and S.-i. Inutsuka, Physica A389, 4580 (2010), arXiv:1006.2663 [astro-ph.HE]

[49] A. El, Z. Xu, and C. Greiner, Phys. Rev. C81, 041901 (2010), arXiv:0907.4500 [hep-ph].

[50] G. S. Denicol, H. Niemi, E. Molnar, and D. H. Rischke, Phys. Rev. D85, 114047 (2012), [Erratum: Phys. Rev.D91,no.3,039902(2015)], arXiv:1202.4551 [nucl-th].

[51] G. S. Denicol, S. Jeon, and C. Gale, Phys. Rev. C90, 024912 (2014), arXiv:1403.0962 [nucl-th]

[52] J. D. Bjorken, Phys. Rev. D 27, 140 (1983).

[53] G. Strang, SIAM J. Numer. Anal. 5, 506 (1968)

[54] J. S. Moreland, J. E. Bernhard, and S. A. Bass, Phys. Rev. C92, 011901 (2015), arXiv:1412.4708 [nucl-th]

[55] W. Ke, J. S. Moreland, J. E. Bernhard, and S. A. Bass, Phys. Rev. C96, 044912 (2017), arXiv:1610.08490 [nuclth],

[56] M. Bluhm, P. Alba, W. Alberico, A. Beraudo, and C. Ratti, Nucl. Phys. A929, 157 (2014), arXiv:1306.6188 [hep-ph].

[57] S. Borsanyi, G. Endrodi, Z. Fodor, S. D. Katz, S. Krieg, C. Ratti, C. Schroeder, and K. K. Szabo, Proceedings, 29th International Symposium on Lattice field theory (Lattice 2011): Squaw Valley, Lake Tahoe, USA, July 10-16, 2011, PoS LATTICE2011, 201 (2011), arXiv:1204.0995 [hep-lat]

[58] F. Karsch, K. Redlich, and A. Tawfik, Phys. Lett. B571, 67 (2003), arXiv:hep-ph/0306208 [hep-ph].

[59] A. Tawfik, Phys. Rev. D71, 054502 (2005), arXiv:hep$\mathrm{ph} / 0412336$ [hep-ph].

[60] T. Hirano, U. W. Heinz, D. Kharzeev, R. Lacey, and Y. Nara, Phys. Lett. B636, 299 (2006), arXiv:nucl- th/0511046 [nucl-th]

[61] C. Nonaka and S. A. Bass, Phys. Rev. C75, 014902 (2007), arXiv:nucl-th/0607018 [nucl-th]

[62] F. Cooper and G. Frye, Phys. Rev. D10, 186 (1974)

[63] S. Pratt and G. Torrieri, Phys. Rev. C82, 044901 (2010), arXiv:1003.0413 [nucl-th].

[64] K. Dusling and T. Schaefer, Phys. Rev. C85, 044909 (2012), arXiv:1109.5181 [hep-ph].

[65] P. Huovinen and H. Petersen, Eur. Phys. J. A48, 171 (2012), arXiv:1206.3371 [nucl-th]

[66] S. A. Bass et al., Prog. Part. Nucl. Phys. 41, 255 (1998) [Prog. Part. Nucl. Phys.41,225(1998)], arXiv:nucl-th/9803035 [nucl-th].

[67] M. Bleicher et al., J. Phys. G25, 1859 (1999), arXiv:hepph/9909407 [hep-ph]

[68] S. Weinberg, Astrophys. J. 168, 175 (1971)

[69] E. Abbas et al. (ALICE), Phys. Lett. B726, 610 (2013), arXiv:1304.0347 [nucl-ex]

[70] J. Adam et al. (ALICE), Phys. Lett. B754, 373 (2016) arXiv:1509.07299 [nucl-ex]

[71] B. Abelev et al. (ALICE), Phys. Rev. C88, 044910 (2013), arXiv:1303.0737 [hep-ex]

[72] K. Aamodt et al. (ALICE), Phys. Rev. Lett. 107, 032301 (2011), arXiv:1105.3865 [nucl-ex].

[73] A. Bilandzic, R. Snellings, and S. Voloshin, Phys. Rev. C83, 044913 (2011), arXiv:1010.0233 [nucl-ex]

[74] E. Shuryak, Physics Letters B 78, 150 (1978)

[75] J. Adam et al. (ALICE), Phys. Lett. B762, 376 (2016), arXiv:1605.02035 [nucl-ex]

[76] L. Landau and E. Lifshitz, The Classical Theory of Fields, 4th ed., Course of Theoretical Physic, Vol. 2 (Butterworth-Heinemann, Oxford, 1980).

[77] V. Greco and C. M. Ko, Phys. Rev. C70, 024901 (2004) arXiv:nucl-th/0402020 [nucl-th]. 